\title{
Cascaded amplifying circuits enable ultrasensitive cellular sensors for toxic metals
}

\author{
Xinyi Wan ${ }^{1,2}$, Francesca Volpetti ${ }^{3}$, Ekaterina Petrova ${ }^{3}$, Chris French ${ }^{1,2}$, Sebastian J. Maerkl $\mathbb{1}^{3}$ and \\ Baojun Wang ${ }^{1,2 \star}$
}

Cell-based biosensors have great potential to detect various toxic and pathogenic contaminants in aqueous environments. However, frequently they cannot meet practical requirements due to insufficient sensing performance. To address this issue, we investigated a modular, cascaded signal amplifying methodology. We first tuned intracellular sensory receptor densities to increase sensitivity, and then engineered multi-layered transcriptional amplifiers to sequentially boost output expression level. We demonstrated these strategies by engineering ultrasensitive bacterial sensors for arsenic and mercury, and improved detection limit and output up to 5,000-fold and 750-fold, respectively. Coupled by leakage regulation approaches, we developed an encapsulated microbial sensor cell array for low-cost, portable and precise field monitoring, where the analyte can be readily quantified via displaying an easy-to-interpret volume bar-like pattern. The ultrasensitive signal amplifying methodology along with the background regulation and the sensing platform will be widely applicable to many other cell-based sensors, paving the way for their real-world applications.

V V hole-cell biosensors have been drawing increasing attention over the last few decades, especially in the rising era of synthetic biology. In contrast to traditional physical and chemical sensors, cellular sensors are renewable, highly selective, easy-to-manufacture and cost-effective ${ }^{1,2}$. Accordingly, bacterial cell-based sensors have been studied for various applications, such as environmental monitoring ${ }^{3-6}$, disease diagnosis ${ }^{7-10}$ and biotherapy $^{11-13}$, bioproduction ${ }^{14}$, mineral surveying ${ }^{15}$ and landmine clearing ${ }^{16}$. Despite their advantages and demonstrated proof-ofconcept success in the laboratory, very few have made it into the market. In addition to biosafety concerns ${ }^{17}$, cellular sensors are often plagued by their unsatisfactory sensing performance that is insufficient to meet the real-world detection requirements, particularly with regard to low sensitivity and limit of detection (LOD) ${ }^{18-20}$, restricted input/output dynamic ranges ${ }^{21,22}$ and lack of field-deployable and easy-to-interpret user interfaces ${ }^{23,24}$.

Recent advances in synthetic biology have provided a number of tools for precise gene expression regulation ${ }^{25}$, such as transcriptional promoter engineering ${ }^{22,26,27}$, translational efficiency tuning $^{21,28}$ and post-translational protein degradation control ${ }^{29,30}$. While some of these have been applied to improving the performance of genetically encoded biosensors ${ }^{20-22,26,27,29}$, most reports only focus on one feature while ignoring others, leading to a trade-off between the different sensing features (for example, improving LOD may lead to high background expression ${ }^{20,22}$, and reducing background expression may reduce maximum output expression and increase $\mathrm{LOD}^{26,29}$ ). In addition, some solutions were case specific (for example, targeting specific promoters ${ }^{26,27}$ ). Consequently, the usage of these sensors is inherently restricted, and a complete yet widely applicable solution for enhancing sensor performance to meet the demands of practical applications is necessary and timely.

Heavy metals contamination of water is a worldwide health issue. For instance, UNICEF has reported that $>140$ million people drink arsenic contaminated water every day ${ }^{31}$. Long-term exposure to arsenic leads to arsenicosis, various skin diseases and cancers.
The traditional laboratory-based assays for metal quantification are time consuming and require specialized trained personnel and expensive equipment. In the predominantly affected resource limited countries, lack of sufficient skilled personnel and healthcare facilities lead people to use contaminated water without testing it. Thus, there is an urgent need to provide simple, affordable, fast onsite sensing solutions for toxic metal contaminated daily resources.

To address these challenges, here we developed a novel, modular sensor signal amplifying methodology, which can rapidly and drastically increase sensitivity and output amplitude of cell-based biosensors. We demonstrated this by engineering and optimizing ultrasensitive Escherichia coli-based sensors for detecting arsenic and mercury water contamination. To reduce background expression accompanying amplified or sensitive sensors, we investigated two approaches to modulate arsenic sensor background level without compromising its detection limit and output dynamic range ${ }^{26,29}$. Further, using the generated sensor variants and hydrogel or microfluidic entrapment, we designed and validated an innovative microbial sensor array that can generate graded volume bar-like patterns in response to varying arsenic pollutant levels. Such easy-to-interpret patterns from the encapsulated sensing array can be simply visualized by a cell phone camera without the need for sophisticated equipment, facilitating its use for portable, low-cost environmental monitoring in the field.

\section{Results}

Modular cascaded amplification of cellular sensor signal. A typical cellular sensor can be abstracted as a three-stage processor comprising a sensing module that recognizes and transduces external signals into intracellular transcriptional signals, a computing module that modulates the transduced sensor signals, and an output actuating module that executes physiological responses (Fig. 1a) ${ }^{1-}$ 3,32 . Our modular signal amplifying methodology integrates three synergistic signal amplification strategies by manipulating the first two modules step-by-step. 
a
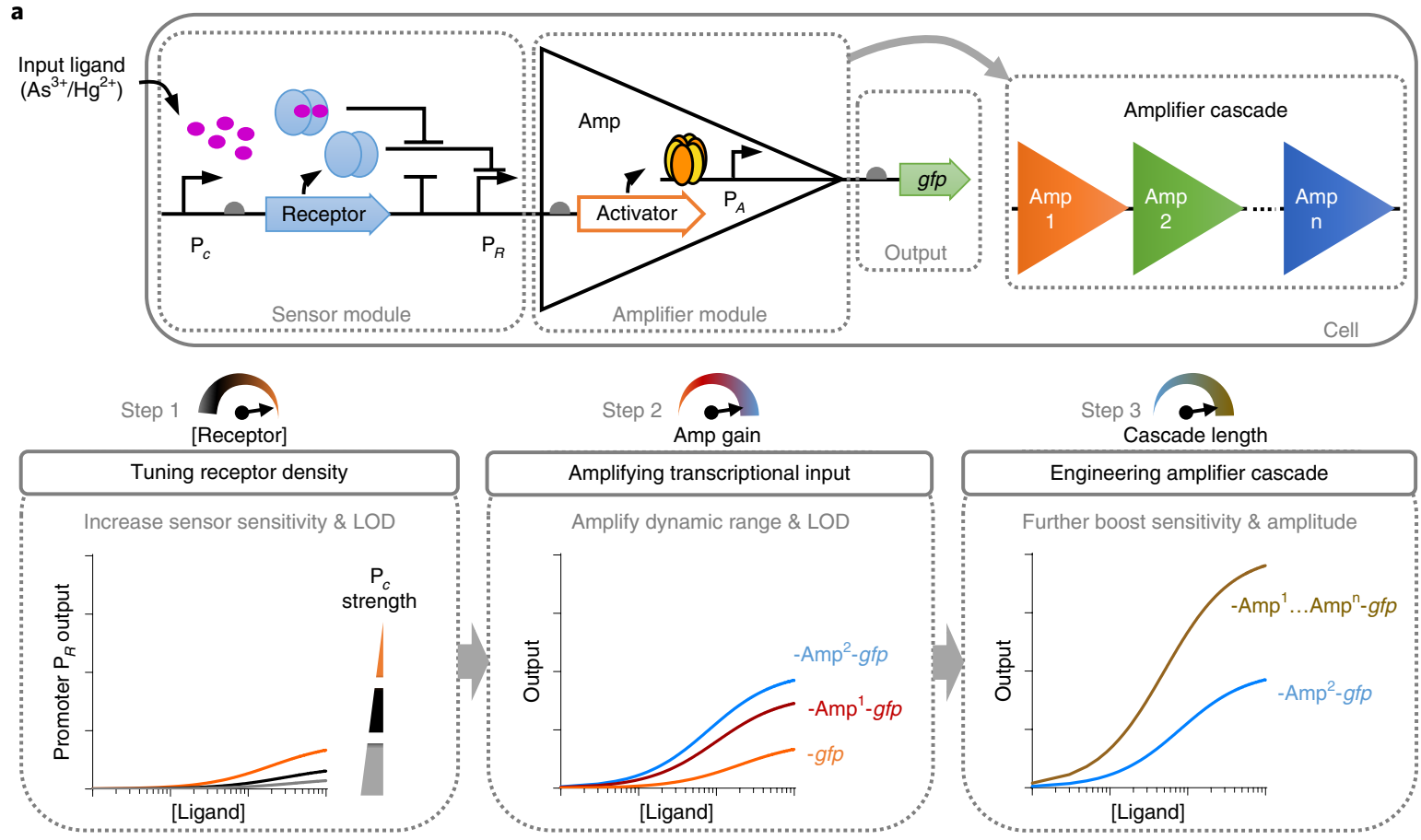

b
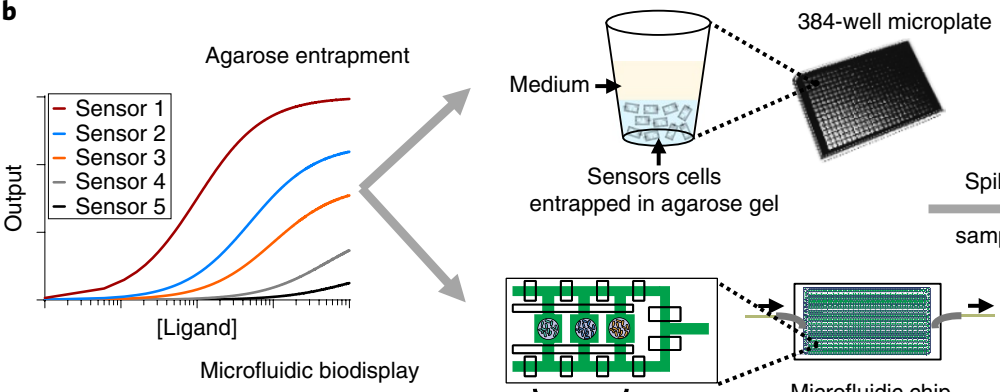
entrapped in agarose gel Spiked with sample water
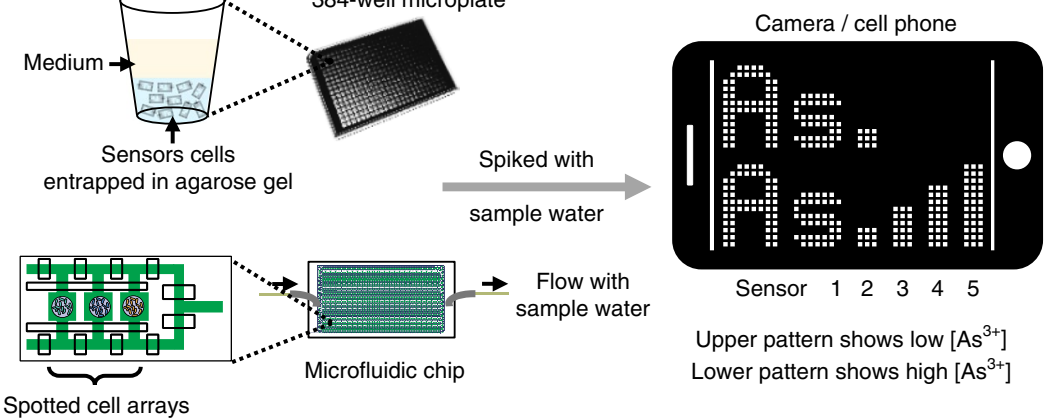

Fig. 1| Modular multilayer signal amplification for engineering ultrasensitive transcription-based cellular sensors. a, Schematic showing a typical repressor-receptor mediated transcription-based sensor in a bacterial cell. The expression of the small-molecule-responsive receptor is driven by a constitutive promoter $\left(P_{C}\right)$. The receptor also acts as a transcriptional repressor, which naturally represses its cognate promoter $\left(P_{R}\right)$. When the target molecules (that is, an input ligand) are present, the receptor binds to its cognate ligand and releases $P_{R^{\prime}}$ which then activates the downstream gene transcription. The first step (Step 1) of the signal amplification approach is to tune the intracellular receptor density by varying the strength of $P_{C}$. For repressor-based sensor modules, weaker $P_{C}$ will lead to lower density of the receptor allowing easier release of $P_{R}$ in the presence of the signal ligand. As illustrated in the lower response diagrams, this results in significant increase of the sensor sensitivity and lowering of the LOD. To expand output dynamic range, a transcriptional amplifier (Amp) is employed to amplify the transduced transcriptional signal from $\mathrm{P}_{R}$ (Step 2). The last step (Step 3) uses a multilayered amplifier cascade, built from cascaded orthogonal amplifiers, to sequentially amplify the transcriptional input signal flow, and thus further boost the sensor's output readout. b. Sensors 1-5 with varying sensitivity and output can be generated by the three-step signal amplification. Using agarose entrapment or microfluidic encapsulation, they are used to build a microbial sensor array that displays an easy-to-read volume bar-like pattern for cell phone-based easyto-use and accurate field monitoring of target environmental contaminants, such as arsenic in drinking water. $P_{A}$, activator's cognate promoter.

The first is tuning intracellular receptor protein density in the sensing module to increase sensor sensitivity (Fig. 1a, Step 1$)^{22}$. Since the sensor's sensitivity is intrinsically tied to the relative concentrations between the receptor and ligand, tuning their respective densities in the cell would lead to improved LOD, and varying the strength of the receptor's constitutive promoter $\left(\mathrm{P}_{C}\right)$ would be a simple and predictable solution. The second is engineering an ultrasensitive activator-based high-gain transcriptional amplifier in the computing module, which amplifies the transduced sensor signal from $\mathrm{P}_{R}$ and hence increase the actuating module's output dynamic range (Fig. 1a, Step 2) ${ }^{20,21}$. The last is cascading multiple orthogonal amplifiers in tandem to amplify the transduced sensor signal and to further boost sensing performance (Fig. 1a, Step 3). The signal amplification enables generating sensor variants of different sensitivities that can be incorporated into encapsulated easy-to-interpret sensor cell arrays for environmental monitoring (Fig. 1b).

Increasing sensor's sensitivity and output dynamic range. We first tested the signal amplifying methodology on a previously studied arsenic sensor (that is, J101-arsR- $\mathrm{P}_{\text {ars }}$ - $g f p$, Supplementary Tables 1 and 2) ${ }^{3}$. This sensor (Fig. 2b) has a constitutive promoter (J101) that drives the expression of an arsenic receptor ArsR, which would de-repress its cognate promoter $\mathrm{P}_{a r s R}$ on arsenite binding and trigger the expression of a reporter gene, $g f p$. However, this sensor can only sense $>10 \mathrm{ppb}$ arsenic (that is, $0.133 \mu \mathrm{M}$ ), so it would not meet the requirement for real applications. 

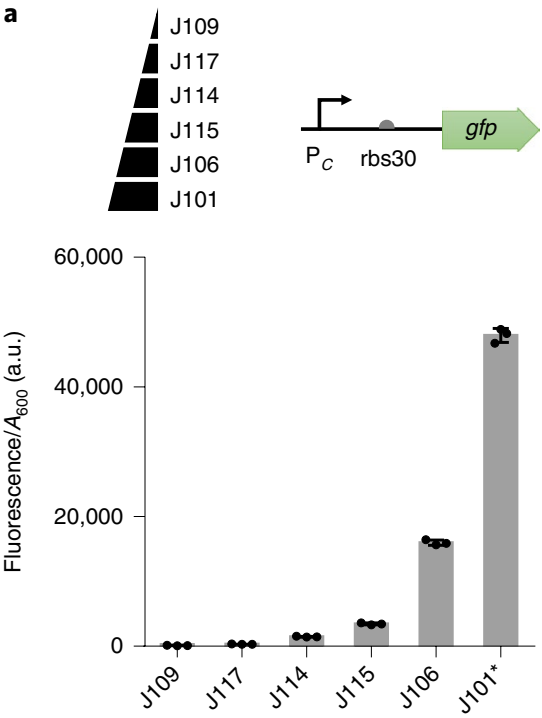

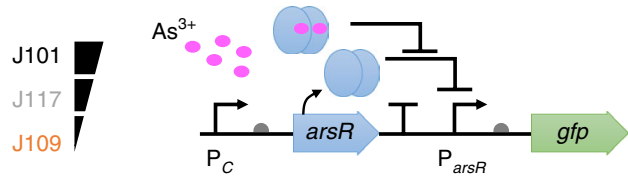

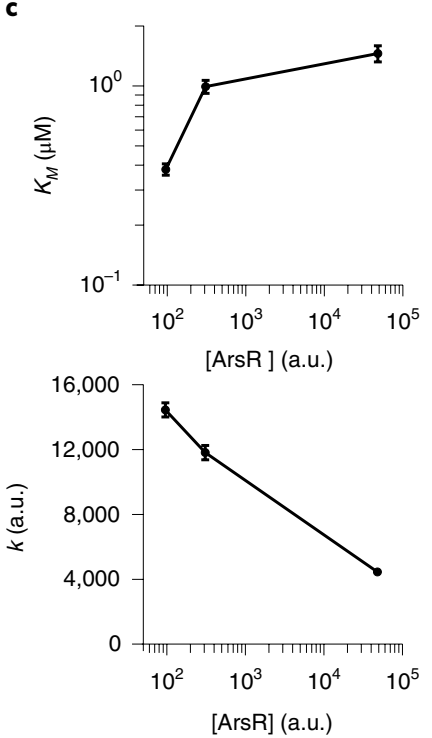

d

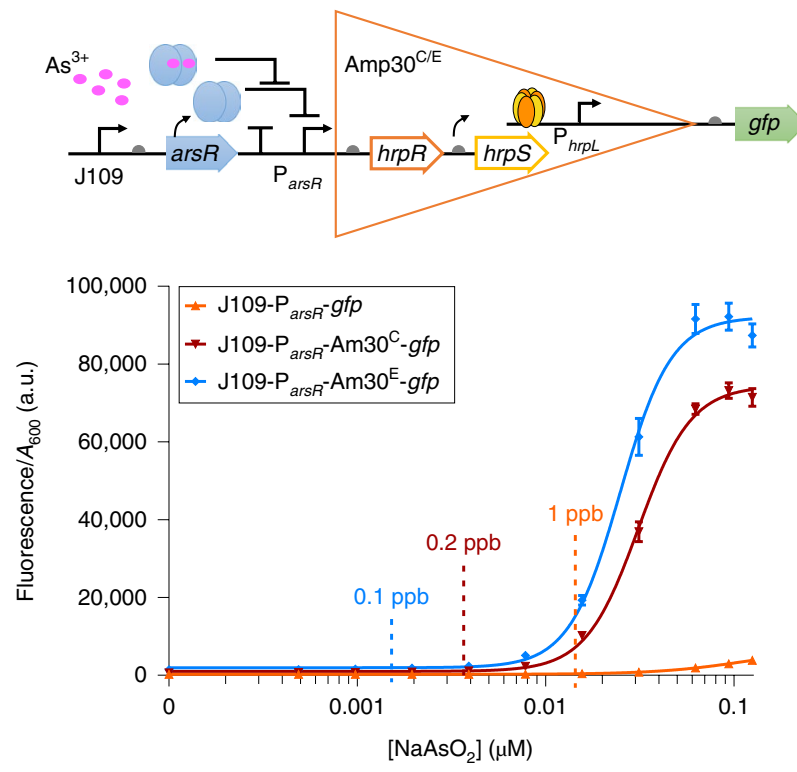

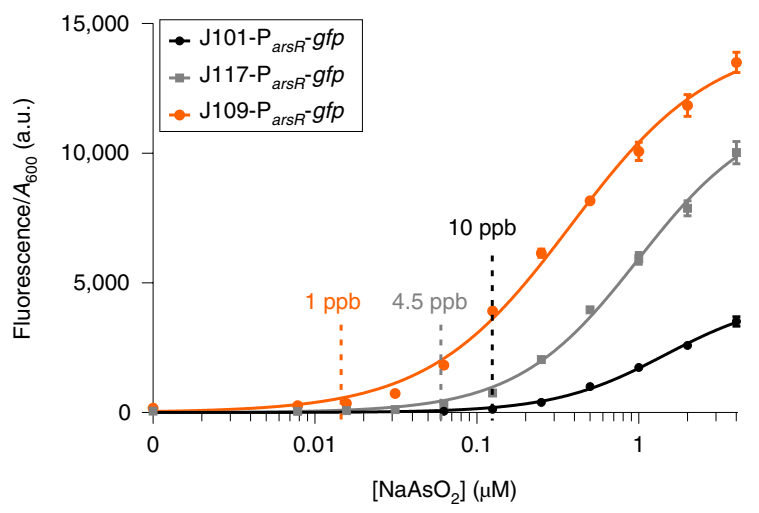

e

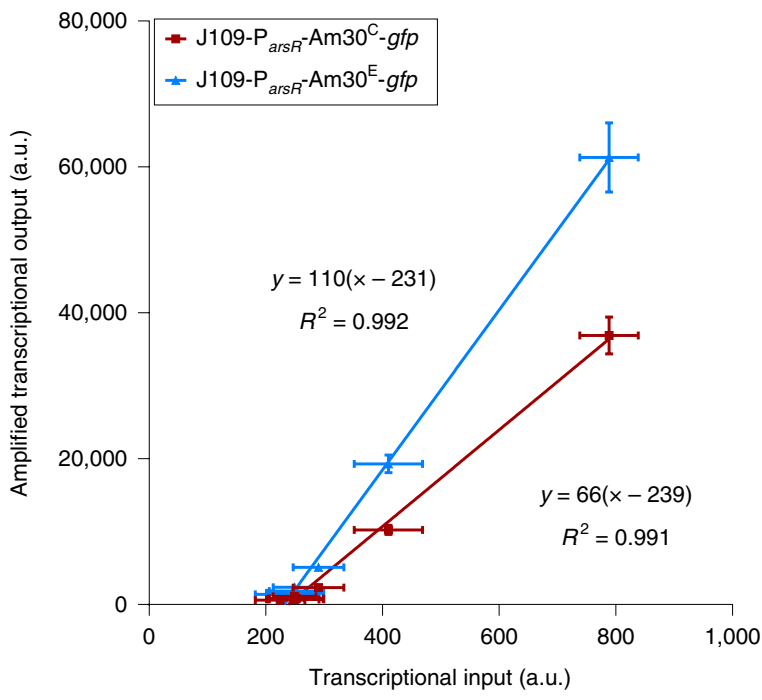

Fig. 2 | Amplifying arsenic sensor by tuning intracellular receptor density and employing single-layer transcriptional amplification. a, Characterization of a set of constitutive promoters $\left(P_{C}\right)$. J101*: rbs32 was used for the J101 promoter. Values are mean \pm s.d. ( $n=3$ biologically independent samples). $\mathbf{b}$, Tuning arsenic receptor ArsR's intracellular density by varying the strength of $P_{C}$, showing that a weaker promoter can improve both sensitivity and output readout of arsenic sensors. Values are mean \pm s.d. ( $n=4$ biologically independent experiments). $\mathbf{c}$, Hill constant $\left(K_{M}\right)$ and the maximum output $(k)$ of the sensor's dose response against the relevant intracellular ArsR concentrations (that is, $\left[\right.$ ArsR], defined by the strength of $P_{C}$, measured by the output fluorescence level of $P_{C}$ shown in a). Values are mean with $95 \%$ confidence intervals $(n=4$ biologically independent experiments). d, Further amplifying the sensitivity and output of arsenic sensors by using a transcriptional amplifier (Amp30 ${ }^{C}$ and $\mathrm{Amp} 30^{\mathrm{E}}$ ), showing improved amplification of optimized amplifier Amp30 . Values are mean \pm s.d. ( $n=4$ biologically independent experiments). e, A scatter plot showing the linear relationships between the non-saturated

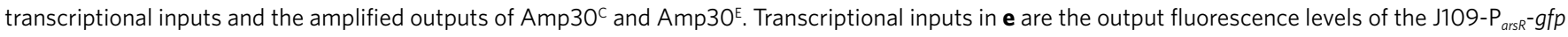
sensor characterized in $\mathbf{d}$. The values shown on the $x$ axis in $\mathbf{e}$ correspond to the fluorescence $/ A_{600}$ values on the $y$ axis in $\mathbf{d}$. Values are mean \pm s.d. $(n=4$ biologically independent experiments). Arsenite $\mathrm{NaAsO}_{2}$ was used in all induction assays. a.u., arbitrary units (fluorescence $/ A_{600}$ ).

To perform the first amplification strategy by tuning the intracellular receptor density, we characterized six constitutive promoters of varying strengths (Fig. 2a), and chose two weak promoters (that is, J117 and J109) to replace J101 in the arsenic sensor. The sensors were then compared under various $\mathrm{NaAsO}_{2}$ induction conditions (Fig. $2 \mathrm{~b}$ and Supplementary Fig. 1). The results showed that the weaker the promoter (that is, the lower the ArsR receptor concentration), the more sensitive and higher the dynamic range of the sensor. That said, there is a limitation on the driving promoter strength as extremely weak promoters may lead to high basal expression and low output dynamic range. Notably, the arsenic sensor with the weakest promoter (J109) could sense $1 \mathrm{ppb}$ arsenic, which is ten times lower than the original sensor.

We fitted the sensors' dose-response curves to a Hill functionbased biochemical model to describe their input-output relationships (see Methods and Supplementary Table 3). In this case, the Hill constant $K_{M}$ is the inducer concentration that provokes halfmaximal activation of a sensor, and $k$ is the sensor's maximum output expression level; therefore, $K_{M}$ is negatively correlated with sensitivity, while $k$ is positively correlated with output amplitude. 
Here, $K_{M}$ showed a four-fold decrease and $k$ showed a three-fold increase from high to low ArsR levels (Fig. 2c), confirming that the arsenic sensor's sensitivity and output amplitude were both increased while the ArsR intracellular concentration was decreased.

To further improve the output expression, we proceeded to introduce a transcriptional amplifier between the sensor module and the reporter. We previously developed a modular amplifier, Amp30', which can amplify transcriptional signal inputs and increase the output readout, but its amplification at low input signal is limited ${ }^{21}$. To achieve optimized high-gain amplification, we retrofitted $A m p 30^{C}$ to generate amplifier $A m p 30^{\mathrm{E}}$ by adopting the consensus $\sigma^{54}$ promoter sequence for its output $\mathrm{P}_{h r p L}$ promoter (Supplementary Fig. 2a), and compared them using the most sensitive arsenic sensor module as the input (that is, J109-arsR- $\mathrm{P}_{\text {ars } R}$ ). Figure $2 \mathrm{~d}$ shows that both amplifiers increased the output expression, and the retrofitted Amp30 $0^{\mathrm{E}}$ performed better than $A m p 30^{\mathrm{C}}$. Notably, the sensor including Amp30 $30^{\mathrm{E}}$ could sense $0.1 \mathrm{ppb}$ arsenic (100-fold improvement of LOD), and increased the output expression level by 440 -fold compared to the original arsenic sensor. We used a simple linear mathematical formula to model the input-output relationship in the linear amplification range for both amplifiers. The results (Fig. 2e) showed a higher amplification gain for Amp30 ${ }^{\mathrm{E}}$ (gain $=110)$ than $A m p 30^{C}$ (gain =66) confirming that the amplifier retrofitting indeed increased the amplification capacity. To analyze the signal fidelity of the amplifiers, we calculated their noise factors across varying transcriptional signal inputs (see Methods). Low noise factors $(<2.5)$ were observed for both Amp30 ${ }^{\mathrm{C}}$ and $\mathrm{Amp} 30^{\mathrm{E}}$ (Supplementary Fig. 2c), indicating that no substantial noise was introduced by the signal amplification and that the enhanced amplifier did not further increase the noise.

Cascaded amplifier further boosts sensitivity and output. In principle, the transduced transcriptional signal of the sensor could be sequentially amplified through multilayer coupled amplifiers. To test this hypothesis, we engineered alternative high-gain transcriptional amplifiers using ultrasensitive phage activator RinA_p80 $\alpha$ (from Staphylococcal aureus phage $80 \alpha)^{33}$ and extracytoplasmic function (ECF) sigma factor ECF11_987 (from Vibrio parahaemolyticus) ${ }^{34}$. An orthogonality characterization was performed, showing no cross activation among the RinA, ECF11 and HrpRS-based amplifiers (Fig. 3a) and hence their potential to be used in one system.

It should be noted that, to realize effective signal amplification, a functional amplifier cascade requires the input-output profile of different coupled amplifiers to be matched and the output signal of an amplifier should be higher than its input signal. Accordingly, we built a library of the RinA/ECF11-based amplifiers of different input/output profiles and amplification capacities (Supplementary Fig. 3). This is achieved by varying the expression level of the underlying activator (RinA/ECF11) in the amplifier through replacing its ribosome binding site and/or addition of a protein degradation tag.

From this library of amplifier variants, we selected Amp31E11 ${ }^{\mathrm{A}}$ as the second layer to be connected to the first layer $A m p 30^{\mathrm{E}}$ to build a two-layer amplifier cascade. To reduce the sensor's basal leakage level and cellular burden, we connected the amplifier cascade to a tight input sensor module (that is, J117-arsR-P $\mathrm{P}_{\text {ars } R}$ ) carried in a low copy number plasmid (that is, pSB4A3-five copies per cell; the previous sensor was in pSB3K3, 10-12 copies per cell) ${ }^{35}$. It was found that the two-layered amplifier (orange curve in Fig. 3b) achieved higher signal output than Amp30 $0^{\mathrm{E}}$ alone (black curve in Fig. 3b) particularly at low arsenic induction levels. In addition, in contrast to the single-layer amplification using Amp30 ${ }^{\mathrm{E}}$ alone, the two-layered amplifier also improved the detection limit by fourfold. Further investigation (by characterizing the Amp $30^{\mathrm{E}}$-amplified sensor alone in low copy pSB4A3) showed that such sensing performance improvement is largely owing to the two-step amplification
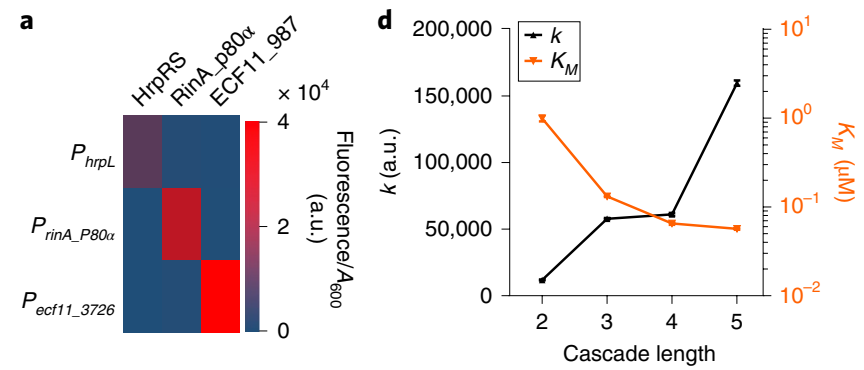

b
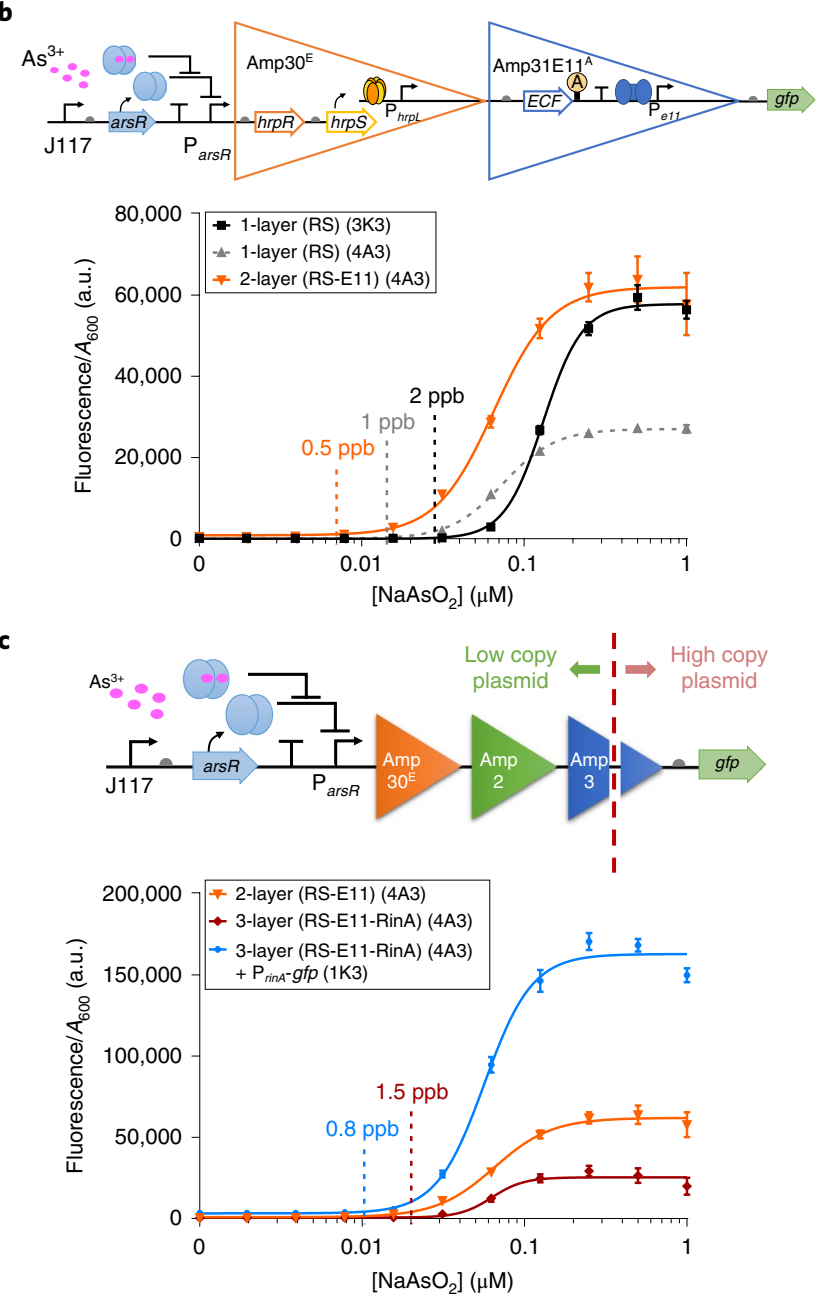

Fig. 3 | Sequential cascaded amplification further boosts the sensor's sensitivity and output amplitude. a, Orthogonality characterization of the three transcriptional amplifiers showing no mutual cross talk. For the assay, $0.25 \mu \mathrm{M} \mathrm{HgCl}_{2}$ was used. Data shown are mean values of three biological replicates, and were collected $8 \mathrm{~h}$ post induction and incubation. $\mathbf{b}$, Design and characterization of an arsenic sensor with a two-layer amplifier cascade, showing improved detection limit and output readout. ' $\mathrm{A}$ ' represents the ASV degradation tag. Values are mean \pm s.d. ( $n=4$ biologically independent experiments). c, Design and characterization of an arsenic sensor with three-layered amplification implemented on two plasmids, showing the output can be further boosted with additional layer of amplification. Values are mean \pm s.d. ( $n=4$ biologically independent experiments). d, Plots showing the Hill constant $\left(K_{M}\right)$ and the maximum output $(k)$ of the sensor's dose response. Values are mean with $95 \%$ confidence intervals $(n=4$ biologically independent experiments). The detailed configurations and plasmid maps of the multi-layered amplifiers are shown in Supplementary Fig. 10. RS, HrpRS-based amplifier. E11, ECF11_987-based amplifier. RinA, RinA_p80 $\alpha$-based amplifier. Arsenite $\mathrm{NaAsO}_{2}$ was used for the induction assays shown in $\mathbf{b}$ and c. a.u., arbitrary units (fluorescence $/ A_{600}$ ). 
whereas the low copy number plasmid may only contribute slightly to the detection limit improvement (gray dashed curve in Fig. 3b).

We next appended a third amplifier, Amp33RinA, to the twolayered amplifier. This three-layer amplified sensor exhibited a poorer dose response (red curve in Fig. 3c) than the two-layer amplified one, possibly due to the amplifiers' incompatible input/ output profiles caused by the load of the amplifier cascade on the host or competition for usage of limited cellular resources. To rescue the amplifying function of the third layer, we shifted the lastlayer amplifier's output promoter along with the reporter to a high copy number plasmid (pSB1K3, >100 copies per cell) (Fig. 3c). We hypothesized that the high copy number plasmid could improve the activator-promoter binding efficiency ${ }^{36}$, amplify the output amplitude and thereby lead to enhanced amplification. The new three-layered amplifier with the output on the high copy number plasmid (blue curve in Fig. 3c) dramatically amplified the output expression and displayed a clearly better performance than the twolayered amplifier. Moreover, the high copy number plasmid further improved the detection limit (from 1.5 to $0.8 \mathrm{ppb}$ ), indicating that the high copy number plasmid may also contribute to shifting or expanding the amplifier's input signal range.

We fitted the sensor dose-response curves to the same aforementioned Hill function model, and found that the Hill constant $K_{M}$ was decreased when the number of amplifiers was increased in the cascade (Fig. $3 \mathrm{~d}$ and Supplementary Table 3). Meanwhile, $k$ was increased, meaning the sensor output expression was stepped up when the transcriptional signal was sequentially amplified by multi-layered amplifiers. These findings confirmed that multi-layered transcriptional amplifiers can further boost a sensor's sensitivity and output amplitude.

Signal amplification modularity and sensor specificity. To verify whether the multi-step amplification method is modular, we swapped the order of the RinA and ECF11-based amplifiers in the previous amplifier cascades (Supplementary Fig. 4). We found that the alternative two- and three-layered amplifiers similarly improved the detection limit and increased the output expression (Supplementary Fig. 4a), as also confirmed by the model-fitted parameters of their dose-response curves (Supplementary Fig. 4b). This result indicates that the multi-step amplification was achieved independently from the type of amplifiers used.

To test the modularity of the entire set of signal amplifying strategies, we next applied them to a second sensor system, the mercury-responsive MerR receptor-based sensor. In contrast to ArsR, MerR is a repressor-activator for its cognate promoter $\mathrm{P}_{\text {merT }}{ }^{37}$. We selected a previously studied mercury sensor (J115-merR- $\mathrm{P}_{\text {merT }}-g f p$, Supplementary Tables 1 and 2$)^{3}$ to perform the optimization. In this sensor, a constitutive promoter (J115) drives the expression of MerR that regulates the activation of $\mathrm{P}_{\text {merT }}$ for $g f p$ output reporter expression. We first replaced the J115 driving promoter with weaker ones, J114 and J109. As expected, this receptor concentration tuning improved the sensor's detection limit (from 50 to $0.3 \mathrm{ppb}$ ) and increased its output dynamic range (Fig. 4a). We then introduced one-, two- and three-layered amplifiers to the most sensitive mercury sensor obtained from receptor density tuning (that is, J109-merR$\left.\mathrm{P}_{\text {merT }}\right)$. Figure $4 \mathrm{~b}$ shows that all amplification variants significantly boosted the sensor's detection limit and output. Compared to the original mercury sensor, our signal amplifying approach improved the detection limit 5,000-fold, and increased the output amplitude 200 -fold at $2 \mathrm{ppb}$ (EPA's safety limit) mercury induction. Similarly, the sensor's fitted dose response showed that its Hill constant $K_{M}$ was decreased dramatically ( $>3$ orders of magnitude) as the number of amplifiers was increased in the amplifier cascade (Fig. 4b). This further demonstrated that ultrasensitive sensors could be achieved using synergistic multi-layered amplification, and that our signal amplifying approach can be applied to different types of sensor.
Additionally, we tested the sensors' specificity against a wide range of metals that could be potential water contaminants. The results (Supplementary Fig. 5) show that the amplified sensors exhibited the same high specificity as their original non-amplified arsenic and mercury sensors ${ }^{3}$. Hence our signal amplification enhanced sensing performance without compromising the sensor specificity.

Tuning sensor's background leakage and output dynamics. We noticed detectable basal expression (leakage) from the characterization of one- and two-layered amplifiers coupled to the most sensitive arsenic sensor module (J109-arsR- $\mathrm{P}_{\text {ars }}$ ) (Fig. 5b,c). Although such leakage may be ignored under certain application circumstances, it could cause issues in other settings, for example, (1) sensitive enzyme-based colorimetric output may easily saturate at high basal expression, restricting titrimetric analysis ${ }^{38}$; (2) narrowing down output dynamic range of the downstream reporter expres$\operatorname{sion}^{39}$ and (3) causing non-stringent side effects for sensor outputs that have therapeutic killing functions.

To reduce sensor basal background, we tested and integrated two different approaches. For the first approach, we inserted an extra ABS downstream of $\mathrm{P}_{\text {ars }}$ to create a 'roadblocking' effect ${ }^{26}$. Additionally, by tuning the distance between the two ABSs (Fig. 5a and Supplementary Table 2), the sensor's leakiness and sensitivity can be adjusted while maintaining the maximum output, leading to modification of the input and output dynamic ranges (Fig. 5b,c).

The second approach uses protease-based post-translational degradation regulation ${ }^{29}$. We first showed that adding a protein degradation tag (AAV) to the reporter protein reduced the output basal expression, but this also significantly lowered the sensor's sensitivity and output level (gray curve in Fig. 5e). To obtain low basal level without sacrificing the high output, we next incorporated the sensor into a TEV protease-based reporter protein degradation control system and used an ECF11-based amplifier to enhance the expression of TEV protease that can cleave the linker between the expressed reporter green fluorescent protein (GFP) and its fused AAV tag (Fig. 5d). To optimize the system, we kept the original $\mathrm{P}_{a r s R}$ in the sensor module to maintain the sensitivity, but used the engineered $\mathrm{P}_{\text {ars }}$ containing double ABSs to achieve tight expression control of the TEV protease. The characterization shows that this design fully protected the GFP reporter from degradation at high arsenic induction levels, while achieving significantly lower basal expression through continuous degradation of the reporter GFP at low arsenic induction levels (orange curve in Fig. 5e). This approach lowered the detection limit from 5 to $0.2 \mathrm{ppb}$, which is similar to that of the original leaky amplified arsenic sensor. In summary, this hybrid regulation system is sufficient to reduce the sensor's basal background while also being able to maintain both the sensor's output amplitude and sensitivity, leading to expanded output dynamic range.

Easy-to-interpret sensor cell array for field monitoring. While investigating our signal amplifying methodology, we generated a set of arsenic sensors of varying detection limits by tuning receptor densities, regulating plasmid copy numbers, $\mathrm{P}_{\text {ars } R}$ engineering and introducing amplifiers or amplifier cascades. Based on these sensors, we sought to design a microbial sensor cell array to facilitate field applications for environmental monitoring, where the analytes can be easily quantified by simply visualizing an easy-to-interpret pattern displayed by the sensor cell array. Moreover, the sensor cell array is designed using agarose hydrogel entrapment ${ }^{40}$ or microfluidic encapsulation ${ }^{23}$, which can mitigate biosafety concerns.

We first selected eight arsenic sensors of increasing detection limits (Supplementary Fig. 6a,b) to test their performance under agarose gel entrapment (see Methods, Supplementary Fig. 6c-e and Supplementary Table 1). The sensors' responses were measured by fluorometry to quantify the fluorescent output (Supplementary Fig. 6d), and an image was subsequently taken using a Gel Doc 


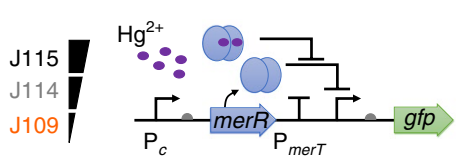

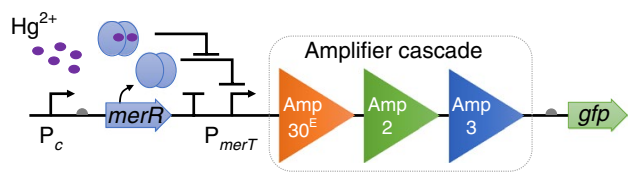
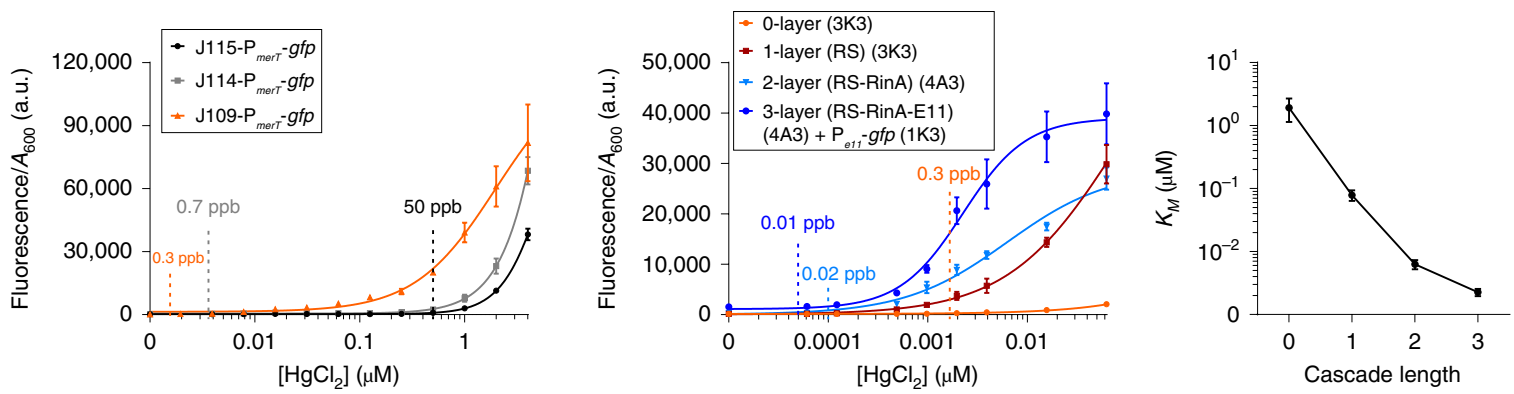

Fig. 4 | Synergistic multi-layered amplification enables ultrasensitive sensors for mercury. a, Design of the mercury-responsive sensor and tuning of MerR receptor intracellular concentration by varying the strength of its constitutive driving promoter $\mathrm{P}_{c}$. Data shown were collected $6 \mathrm{~h}$ post induction and incubation. Values are mean \pm s.d. ( $n=3$ biologically independent samples). $\mathbf{b}$, Design and characterization of mercury sensor with multi-layered amplification. Top panel: a schematic of mercury sensor with multi-layered amplifiers. Detailed configurations of the multi-layered amplifiers are shown in Supplementary Fig. 10. Bottom left panel: mercury sensor's dose response by using a different number of layers of amplification. Values are mean \pm s.d. ( $n=3$ biologically independent samples). Bottom right panel: Hill constant $\left(K_{M}\right)$ of the sensors' fitted dose response against different amplifier cascade lengths. Values are mean with $95 \%$ confidence intervals ( $n=3$ biologically independent samples). a.u., arbitrary units.

a

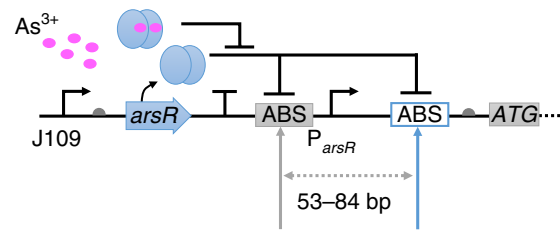

Native ABS Extra ABS b

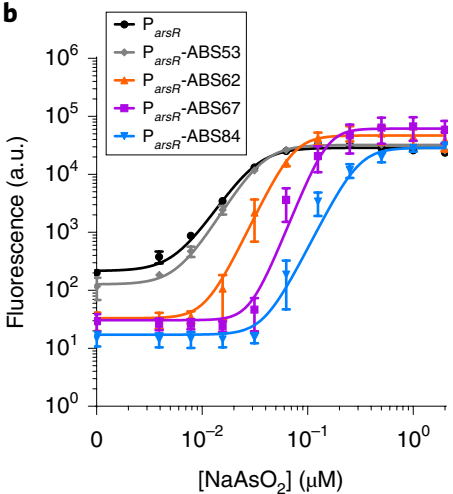

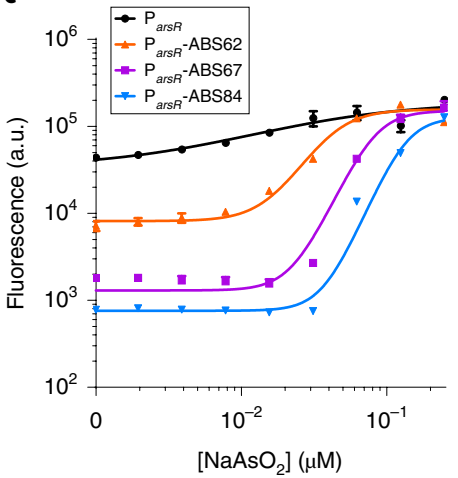

d

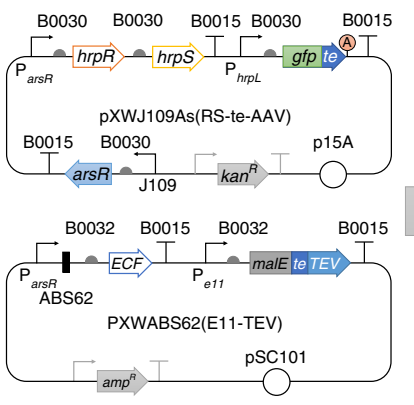

Continuous degradation

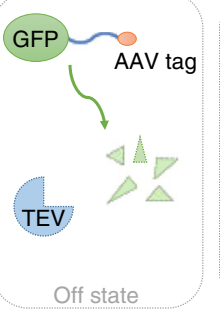

Regulated degradation

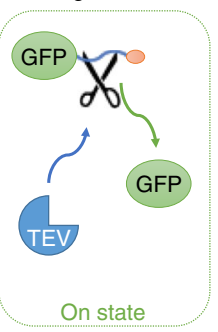

e

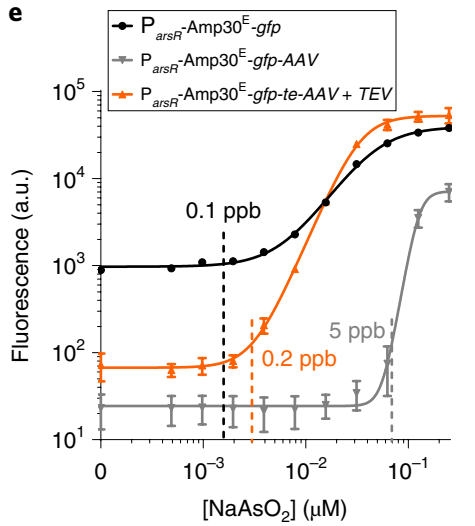

Fig. 5 | Tuning the sensor background and output dynamic range via promoter engineering and reporter degradation regulation. a, Engineering arsenic promoters by inserting an extra ArsR binding site (ABS) downstream of $P_{\text {arse. }}$. The distance between the two ABSs varies between 53 and 84 base pairs. b,c, Dose responses of engineered arsenic sensors as in $\mathbf{a}$, showing that double ABSs can reduce the background output of arsenic sensor (J109-P $P_{\text {ars }}$ sensor module) with a one- (b) or two-layered (c) amplifier, while maintaining the maximum output levels. d, Schematic showing protease-mediated regulation of the background and output dynamic range for an arsenic sensor. ' $A$ ' represents the $A A V$ degradation tag. Off state: when there is no $\mathrm{NaAs} \mathrm{O}_{2}$ induction. On state: when there is $\mathrm{NaAsO}_{2}$ induction. e, Dose responses of the arsenic sensor as in $\mathbf{d} 8 \mathrm{~h}$ post induction and incubation. Black curve represents the sensor without AAV tag. Gray curve represents the sensor with AAV tag not cleavable by TEV protease. Orange curve represents the genetic circuit shown in $\mathbf{d}$, in which the AAV tag can be cleaved off by TEV protease. Values in $\mathbf{b}, \mathbf{c}, \mathbf{e}$ are mean \pm s.d. of median values from flow cytometry assays ( $n=3$ biologically independent experiments). a.u., arbitrary units. 


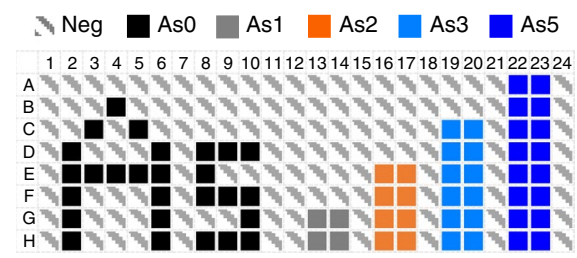

b

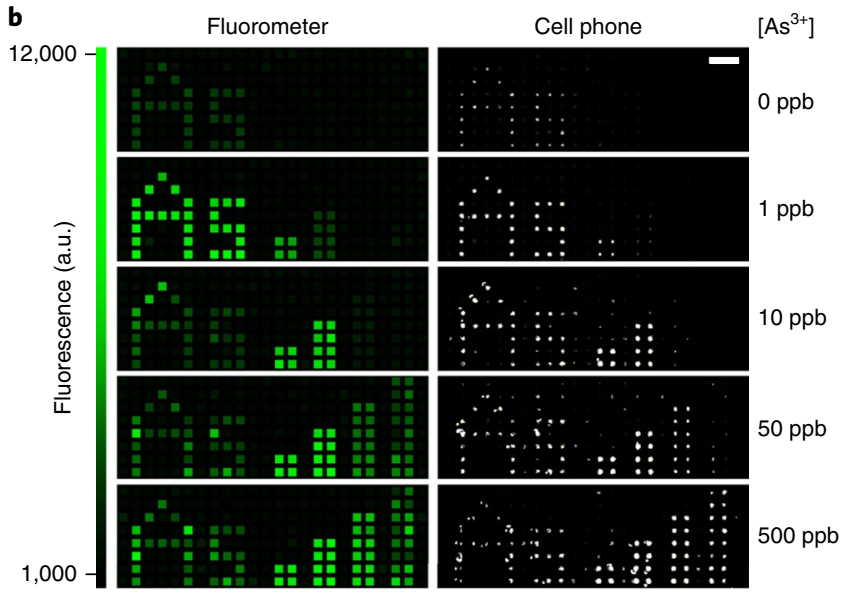

c

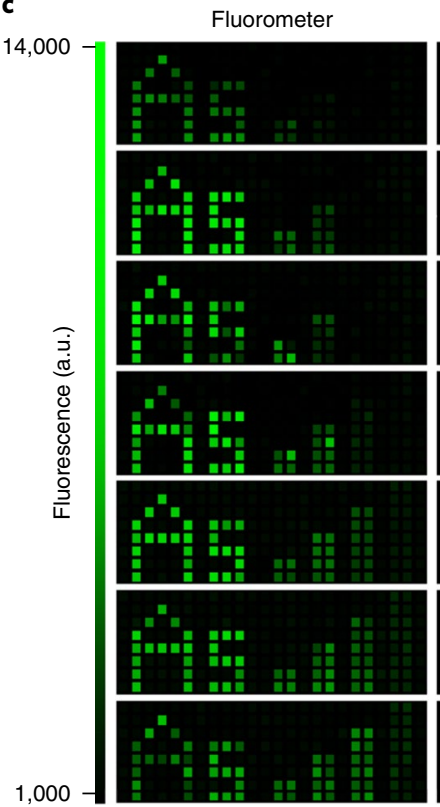

Cell phone

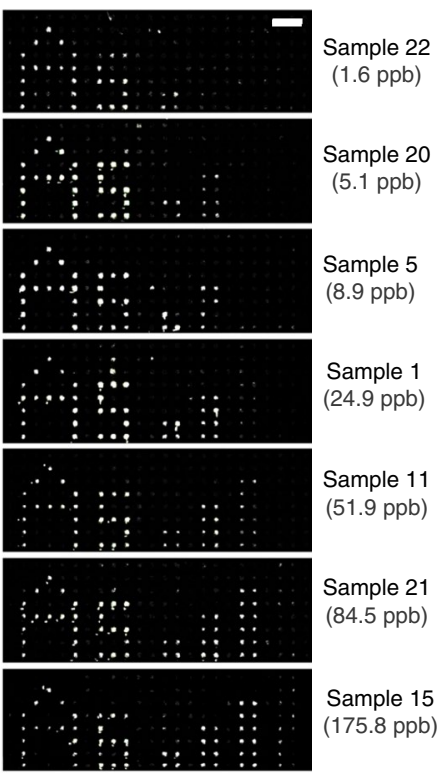

d

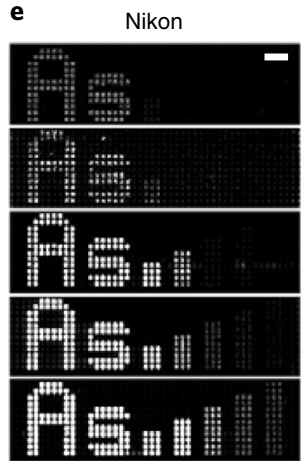

USB

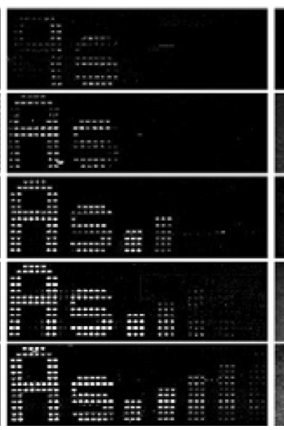

Cell phone

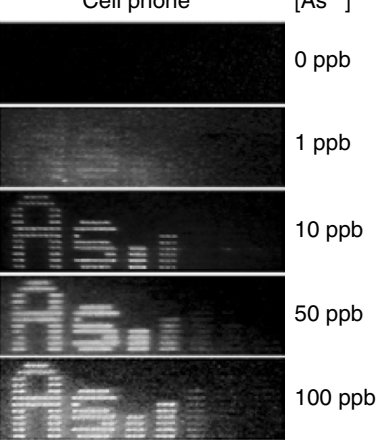

Fig. 6 | Microbial sensor array display enabled by agarose hydrogel entrapment and microfluidic encapsulation for easy-to-use monitoring of arsenic contamination. a, Design of the sensor array in a half 384-well microplate with eight rows (A-H) and 24 columns (1-24). 'As' symbol represents arsenic for identifying the type of contamination, and the number of volume bars indicates the relevant arsenic concentration. $\mathbf{b}$, Agarose gel encapsulationenabled microbial sensor array for monitoring arsenic contamination, showing different output response patterns on various arsenic induction levels $24 \mathrm{~h}$ post incubation. These experiments were repeated four times independently with similar results. Left panel: fluorometry data. Right panel: images taken by a cell phone camera. Scale bar, $1 \mathrm{~cm}$. c, Agarose gel encapsulation-enabled microbial sensor array for monitoring arsenic contamination from groundwater samples. The water samples were mixed with an optimized MOPS medium (with $1 / 4 \mathrm{PO}_{4}{ }^{3-}$ ) at a 1:1 ratio. The arsenic concentrations after two times dilution are shown in brackets. These experiments were repeated twice independently with similar results. Scale bar, $1 \mathrm{~cm}$. $\mathbf{d}$, Design of the arsenic sensor array based on microfluidic biodisplay comprising 16 rows and 48 columns. e, Microfluidic encapsulation-enabled sensor array display for monitoring arsenic levels. Left panel: images acquired by a Nikon microscope. These experiments were repeated twice independently with similar results. Middle panel: images acquired by a FITC USB fluorescence microscope. Right panel: images taken by a cell phone camera. Scale bar, 1 mm. a.u., arbitrary units.

imaging system to visualize the output (Supplementary Fig. 6e). The fluorometry measurement shows that the gel-entrapped sensors exhibited a similar order of sensitivities as those in liquid culture (Supplementary Fig. 6a,b). However, due to the lower sensitivity of the gel doc imaging camera, some sensors (for example, As4) appeared less sensitive in the image taken by the camera (Supplementary Fig. 6e).

We next designed the sensor cell array entrapped in agarose gel using a subset of the eight characterized arsenic sensors (As0-3 and As5) that were spotted in a 384-well microplate following a layout designed to display a volume bar-like pattern (Fig. 6a). We tested the sensor cell array under various arsenic induction levels after $24 \mathrm{~h}$ incubation. Here, a cell phone camera was used to simplify the imaging procedure in addition to the fluorometric assay (see Methods). Expected volume bar-like fluorescent patterns were observed from both the cell phone image and the fluorometric measurement; that is, no arsenic-no pattern, $1 \mathrm{ppb}$ - 'As' with 1 bar, $10 \mathrm{ppb}$-'As' with two bars, $50 \mathrm{ppb}$ - 'As' with three bars and $500 \mathrm{ppb}$-'As' with four bars (Fig. 6b). Three further repeats were performed on different days with similar results obtained (Supplementary Fig. 6f), demonstrating the high stability of the agarose hydrogel-entrapped sensor cell array. Additionally, we observed stronger output fluorescent signals after longer incubation time (Supplementary Fig. 6f) and the sensor cell array can also work at room temperature (Supplementary Fig. $6 \mathrm{~g}$ ).

To demonstrate the functionality of our arsenic sensors in the real environment, we used our sensor cell arrays to report arsenic contamination in groundwater samples collected from Bangladesh (see Methods, Fig. 6c and Supplementary Table 4). We considered that arsenate could be the predominant arsenic species in the 
water samples, and cells might have failed to respond to arsenate due to high levels of phosphate in the M9 medium (Supplementary Fig. 7a-f) and arsenate shares the same cellular uptake system as phosphate $^{41}$. Therefore, a modified MOPS rich medium was used (Supplementary Fig. 7h,j-1,o, see Methods). This maximized the sensors' responses to arsenate, and allowed our engineered sensors and the sensor cell arrays to robustly monitor real environmental samples (Fig. 6c).

Finally, we tested our sensor cell array on a recently developed portable microfluidic biodisplay platform ${ }^{23}$ (see Methods, Fig. 6d,e and Supplementary Fig. 6i). The microfluidic device contains 768 individually programmable biopixels and each biopixel contains a chamber where different sensor cells can be spotted. Each chamber has valves around it to control the flow of media or samples to the sensors, and also to entrap the sensor cells. Similar results of an easy-to-interpret volume bar-like pattern were obtained from a Nikon fluorescence microscope and portable devices including both a low-cost USB microscope and a cell phone camera (Fig. 6e and Supplementary Fig. 6j).

\section{Discussion}

We developed a modular cascaded signal amplifying methodology and combined it with basal background tuning approaches to provide an integrated solution for improving the sensitivity and output dynamic range of cell-based sensors. Using this methodology, we drastically increased the sensitivity of the two exemplar arsenic and mercury sensors with detection limits of $\leq 0.1$ and $\leq 0.01 \mathrm{ppb}$, respectively, and improved output readout 750 - and 200-fold when detecting arsenic or mercury, respectively, at guideline limit values. Owing to its modularity and simplicity, the presented method can be applied to improve many other cell-based and potentially cellfree genetically encoded sensors ${ }^{42,43}$ for a broad range of real-world applications, including environmental assessment ${ }^{3-6}$, disease diagno$\operatorname{sis}^{7-10,44,45}$, bioproduction ${ }^{14,46}$, mining and detection of landmines ${ }^{15,16}$. In particular, the study will aid the development of a new generation of field-deployable, low-cost and easy-to-use biosensors for heavy metal water contamination in the field; a worldwide health issue.

We showed for the first time that multiple layers of transcriptional amplifiers can in-tandem amplify a transduced sensor signal in vivo. These engineered high-gain amplifiers and amplifier cascades did not introduce an obvious response delay (Supplementary Fig. 4c) or notable noise during signal amplification (Supplementary Figs. $2 \mathrm{c}$ and 8 ), which are important for modulating biological signals due to their inherent slow dynamics and noisy characteristics. Moreover, they did not show any notable toxicity to host cells when induced by target pollutants inside their WHO/EPA safety limit levels (Supplementary Fig. 9), indicating that the metabolic load of our amplifier constructs was compliant to common $E$. coli host strains. We noted that our amplifiers not only increased the coupled sensor's output level, but also improved its detection limit (Figs. 2d, $3 \mathrm{~b}$ and $4 \mathrm{~b}$ and Supplementary Fig. 4a). We consider that this could be due to the mode of action of transcription in nature. As transcription events occur in bursts ${ }^{47}$, the transduced transcription bursts at low level of input can be captured and amplified by the downstream ultrasensitive transcriptional amplifiers, thus leading to detectable output reporter expression and lowering of the sensor detection limit. This assumption seems consistent with the two cell populations observed in the flow cytometry assays (Supplementary Figs. 1,2 and 8).

In addition, two approaches were investigated and combined to address the issue of sensor background leakage. They can modulate the sensors' leakage level, sensitivity and dynamic ranges, as well as lower the sensor basal background without reducing the maximum output level. Although more complex, the protease-based reporter degradation control approach should be broadly applicable to different types of input sensor to regulate any protein levels in the amplifier cascade.
Finally, using the engineered arsenic sensor variants generated by the signal amplification and the leakage regulation, we developed a microbial sensor array to display an intuitive volume bar-like pattern to indicate the cognate pollutant level in environmental water samples. The sensor array's output patterns can be simply captured by a cell phone camera without the need for sophisticated equipment, facilitating its potential use as a portable, low-cost environmental monitoring tool in the field. The present detection range of the arsenic sensor array can be further expanded or refined by varying the number and types of arsenic sensor variant. Furthermore, the sensor cell array can distinguish true negative responses from false negative responses caused by toxic components in water samples (Supplementary Fig. 6h). Although the current sensor cell array requires $24 \mathrm{~h}$ of incubation to generate sufficient fluorescence output for visualization, further optimization can be done to reduce the processing time if needed. For example, cell densities can be optimized to increase fluorescence levels such that the output can rapidly reach the visual detection threshold, and the use of fast enzymatic reaction-based outputs (for example, NanoLuc ${ }^{44}$ or LacZ $\alpha$ peptide ${ }^{48}$ ) could speed up the detection response. In contrast to typical sensors having a single colorimetric output, our sensor cell array makes it easier and faster for end users to tell the type and level of the cognate contaminant in the sample, and could be readily adapted to other cellular biosensors. By entrapping sensor cells inside hydrogels or microfluidic devices, it notably reduces the chance of their escape to the open environment and helps mitigate the biosafety concerns for their final field application. With the new advances in cell entrapping materials and preserving methods, the engineered microbial sensor array has the potential to be stably stored for long-term use $\mathrm{u}^{23,40,49}$.

\section{Online content}

Any methods, additional references, Nature Research reporting summaries, source data, statements of data availability and associated accession codes are available at https://doi.org/10.1038/ s41589-019-0244-3.

\section{Received: 23 August 2018; Accepted: 11 February 2019;} Published online: 25 March 2019

\section{References}

1. van der Meer, J. R. \& Belkin, S. Where microbiology meets microengineering: design and applications of reporter bacteria. Nat. Rev. Microbiol. 8, 511-522 (2010)

2. Kim, H. J., Jeong, H. \& Lee, S. J. Synthetic biology for microbial heavy metal biosensors. Anal. Bioanal. Chem. 410, 1191-1203 (2018).

3. Wang, B., Barahona, M. \& Buck, M. A modular cell-based biosensor using engineered genetic logic circuits to detect and integrate multiple environmental signals. Biosens. Bioelectron. 40, 368-376 (2013).

4. Stocker, J. et al. Development of a set of simple bacterial biosensors for quantitative and rapid measurements of arsenite and arsenate in potable water. Environ. Sci. Technol. 37, 4743-4750 (2003).

5. De Mora, K. et al. A pH-based biosensor for detection of arsenic in drinking water. Anal. Bioanal. Chem. 400, 1031-1039 (2011).

6. Cao, Y. et al. Programmable assembly of pressure sensors using patternforming bacteria. Nat. Biotechnol. 35, 1087-1093 (2017).

7. Riglar, D. T. et al. Engineered bacteria can function in the mammalian gut long-term as live diagnostics of inflammation. Nat. Biotechnol. 35, 653-658 (2017).

8. Mimee, M. et al. An ingestible bacterial-electronic system to monitor gastrointestinal health. Science 360, 915-918 (2018).

9. Courbet, A. et al. Detection of pathological biomarkers in human clinical samples via amplifying genetic switches and logic gates. Sci. Transl. Med. 7, 289ra83 (2015).

10. Watstein, D. M. \& Styczynski, M. P. Development of a pigment-based whole-cell zinc biosensor for human serum. ACS Synth. Biol. 7, 267-275 (2018)

11. Duan, F. \& March, J. C. Engineered bacterial communication prevents Vibrio cholerae virulence in an infant mouse model. Proc. Natl Acad. Sci. USA 107, 11260-11264 (2010)

12. Hwang, I. Y. et al. Reprogramming microbes to be pathogen-seeking killers. ACS Synth. Biol. 3, 228-237 (2014). 
13. Ho, C. L. et al. Engineered commensal microbes for diet-mediated colorectal-cancer chemoprevention. Nat. Biomed. Eng. 2, 27-37 (2018).

14. Zhang, F., Carothers, J. M. \& Keasling, J. D. Design of a dynamic sensorregulator system for production of chemicals and fuels derived from fatty acids. Nat. Biotechnol. 30, 354-359 (2012).

15. Cerminati, S., Soncini, F. C. \& Checa, S. K. Selective detection of gold using genetically engineered bacterial reporters. Biotechnol. Bioeng. 108, 2553-2560 (2011).

16. Belkin, S. et al. Remote detection of buried landmines using a bacterial sensor. Nat. Biotechnol. 35, 308-310 (2017)

17. Dana, G. V., Kuiken, T., Rejeski, D. \& Snow, A. A. Synthetic biology: four steps to avoid a synthetic-biology disaster. Nature 483, 29-29 (2012).

18. Shemer, B., Koshet, O., Yagur-Kroll, S. \& Belkin, S. Microbial bioreporters of trace explosives. Curr. Opin. Biotechnol. 45, 113-119 (2017).

19. Landry, B. P. et al. Phosphatase activity tunes two-component system sensor detection threshold. Nat. Commun. 9, 1433 (2018).

20. Kim, H. J. et al. Development of a highly specific and sensitive cadmium and lead microbial biosensor using synthetic CadC-T7 genetic circuitry. Biosens. Bioelectron. 79, 701-708 (2016)

21. Wang, B., Barahona, M. \& Buck, M. Engineering modular and tunable genetic amplifiers for scaling transcriptional signals in cascaded gene networks. Nucleic Acids Res. 42, 9484-9492 (2014).

22. Wang, B., Barahona, M. \& Buck, M. Amplification of small moleculeinducible gene expression via tuning of intracellular receptor densities. Nucleic Acids Res. 43, 1955-1964 (2015).

23. Volpetti, F., Petrova, E. \& Maerkl, S. J. A microfluidic biodisplay. ACS Synth. Biol. 6, 1979-1987 (2017).

24. Prindle, A. et al. A sensing array of radically coupled genetic 'biopixels' Nature 481, 39-44 (2012).

25. Ang, J. et al. Tuning response curves for synthetic biology. ACS Synth. Biol. 2, 547-567 (2013)

26. Merulla, D. \& van der Meer, J. R. Regulatable and modulable background expression control in prokaryotic synthetic circuits by auxiliary repressor binding sites. ACS Synth. Biol. 5, 36-45 (2016).

27. Chen, Y. et al. Tuning the dynamic range of bacterial promoters regulated by ligand-inducible transcription factors. Nat. Commun. 9, 64 (2018).

28. Salis, H. M., Mirsky, E. A. \& Voigt, C. A. Automated design of synthetic ribosome binding sites to control protein expression. Nat. Biotechnol. 27, 946-950 (2009).

29. Fernandez-Rodriguez, J. \& Voigt, C. A. Post-translational control of genetic circuits using Potyvirus proteases. Nucleic Acids Res. 44, 6493-6502 (2016).

30. Cameron, D. E. \& Collins, J. J. Tunable protein degradation in bacteria. Nat. Biotechnol. 32, 1276-1281 (2014).

31. Ravenscroft, P. Predicting the Global Extent of Arsenic Pollution of Groundwater and its Potential Impact on Human Health (UNICEF, New York, 2007)

32. Wang, B. \& Buck, M. Customizing cell signaling using engineered genetic logic circuits. Trends Microbiol. 20, 376-384 (2012).

33. Quiles-Puchalt, N. et al. A super-family of transcriptional activators regulates bacteriophage packaging and lysis in Gram-positive bacteria. Nucleic Acids Res. 41, 7260-7275 (2013)

34. Rhodius, V. A. et al. Design of orthogonal genetic switches based on a crosstalk map of $\sigma$ s, anti- $\sigma$ s, and promoters. Mol. Syst. Biol. 9, 702 (2013).

35. Liu, Q. et al. Orthogonality and burdens of heterologous and gate gene circuits in E. coli. ACS Synth. Biol. 7, 553-564 (2018).

36. Potvin-Trottier, L., Lord, N. D., Vinnicombe, G. \& Paulsson, J. Synchronous long-term oscillations in a synthetic gene circuit. Nature 538, 514-517 (2016).

37. Chang, C. C. et al. Structural basis of the mercury(II)-mediated conformational switching of the dual-function transcriptional regulator MerR. Nucleic Acids Res. 43, 7612-7623 (2015).

38. Wackwitz, A. et al. Internal arsenite bioassay calibration using multiple bioreporter cell lines. Microb. Biotechnol. 1, 149-157 (2008).
39. Nielsen, A. A. K. et al. Genetic circuit design automation. Science 352, aac7341 (2016).

40. Buffi, N. et al. Development of a microfluidics biosensor for agarose-bead immobilized Escherichia coli bioreporter cells for arsenite detection in aqueous samples. Lab Chip 11, 2369-2377 (2011).

41. Willsky, G. R. \& Malamy, M. H. Effect of arsenate on inorganic phosphate transport in Escherichia coli. J. Bacteriol. 144, 366-374 (1980).

42. Pardee, K. et al. Rapid, low-cost detection of Zika virus using programmable biomolecular components. Cell 165, 1255-1266 (2016).

43. Rampley, C. P. N. et al. Development of simcells as a novel chassis for functional biosensors. Sci. Rep. 7, 7261 (2017).

44. Cevenini, L. et al. A novel bioluminescent NanoLuc yeast-estrogen screen biosensor (nanoYES) with a compact wireless camera for effect-based detection of endocrine-disrupting chemicals. Anal. Bioanal. Chem. 410 1237-1246 (2018)

45. Wen, K. Y. et al. A cell-free biosensor for detecting quorum sensing molecules in P. aeruginosa-infected respiratory samples. ACS Synth. Biol. 6, 2293-2301 (2017).

46. Nikel, P. I., Martínez-García, E. \& De Lorenzo, V. Biotechnological domestication of pseudomonads using synthetic biology. Nat. Rev. Microbiol. 12, 368-379 (2014).

47. Choi, P. J., Cai, L., Frieda, K. \& Xie, X. S. A stochastic single-molecule event triggers phenotype switching of a bacterial cell. Science 322, 442-446 (2008).

48. Ma, D. et al. Low-cost detection of norovirus using paper-based cell-free systems and synbody-based viral enrichment. Synth. Biol. 3, ysy018 (2018).

49. Liu, X. et al. 3D printing of living responsive materials and devices. Adv. Mater. 30, 107821 (2018).

\section{Acknowledgements}

We thank M. Billah (Khulna University) and his colleagues for facilitating our collections of groundwater samples in Bangladesh. This work was supported by UK BBSRC project grant (no. BB/N007212/1), Leverhulme Trust research grant (no. RPG-2015-445), Wellcome Trust Seed Award in Science (no. 202078/Z/16/Z) and EPSRC/BBSRC Global Challenges Research Fund Awards. X.W. was supported by scholarships from the China Scholarship Council and Scottish Universities Life Sciences Alliance. F.V., E.P. and S.J.M. were supported by the Ecole Polytechnique Federale de Lausanne, a Swiss National Science Foundation Grant (no. CR23I2 140697) and a SystemsX.ch Special Opportunity Grant (no. 2015/325).

\section{Author contributions}

B.W. conceived and led the study. X.W. designed the experiments with inputs and supervision from B.W. and C.F. X.W. performed all the experiments and data analysis excluding the microfluidics-based experiments. F.V., E.P. and S.J.M. designed and performed the microfluidics-based experiments. All authors took part in the interpretation of results and preparation of materials for the manuscript. B.W. and X.W. wrote the manuscript with comments from all authors.

\section{Competing interests}

B.W. and X.W. filed a patent application based on the technology invention in this work.

\section{Additional information}

Supplementary information is available for this paper at https://doi.org/10.1038/ s41589-019-0244-3.

Reprints and permissions information is available at www.nature.com/reprints. Correspondence and requests for materials should be addressed to B.W. Publisher's note: Springer Nature remains neutral with regard to jurisdictional claims in published maps and institutional affiliations.

(c) The Author(s), under exclusive licence to Springer Nature America, Inc. 2019 


\section{Methods}

Strains, plasmids and growth conditions. Plasmid cloning and genetic circuit characterization were all performed in E. coli TOP10. Cells were cultured in lysogeny broth medium $\left(10 \mathrm{gl}^{-1}\right.$ peptone, $5 \mathrm{gl}^{-1} \mathrm{NaCl}, 5 \mathrm{gl}^{-1}$ yeast extract), with appropriate antibiotics. The antibiotic concentrations used were $50 \mu \mathrm{gll}^{-1}$ for both kanamycin and ampicillin, and $25 \mathrm{\mu g} \mathrm{ml}^{-1}$ for chloramphenicol. For circuit characterization, the engineered bacteria were first inoculated from a single colony on a freshly streaked solid lysogeny broth plate to $2 \mathrm{ml}$ lysogeny broth medium, and cultured overnight at $37^{\circ} \mathrm{C}$ with shaking ( 200 r.p.m.). Then the cells were diluted 100 -fold from the overnight culture into fresh lysogeny broth medium. For liquid culture induction, the diluted culture was loaded into a 96-well microplate (655096, Greiner Bio-One) and induced with $5 \mu \mathrm{l}$ inducers to a final volume of $200 \mu \mathrm{l}$ per well. For testing the constitutive promoters, $200 \mu \mathrm{l}$ of diluted culture were loaded to each well. The microplate was sealed with an air permeable film (AXY2006, SLS), and incubated in a shaker incubator (AS-03020-00, Medical Supply Co. Ltd) with continuous shaking ( 1,000 r.p.m., $\left.37^{\circ} \mathrm{C}\right)$. After $5 \mathrm{~h}$, unless otherwise indicated, a fluorescence plate reader (BMG FLUOstar) was used to monitor cell growth and measure fluorescence.

Antibiotics and other reagents used for induction assays (that is, $\mathrm{HgCl}_{2}$ $\mathrm{NaAsO}_{2}, \mathrm{Na}_{2} \mathrm{HAsO}_{4}, \mathrm{CuSO}_{4}, \mathrm{MgSO}_{4}, \mathrm{ZnCl}_{2}, \mathrm{FeCl}_{2}, \mathrm{NiCl}_{2}, \mathrm{MnCl}_{2}, \mathrm{PbCl}_{2}, \mathrm{CoCl}_{2}$ and $\mathrm{CdCl}_{2}$ ) were analytical grade and purchased from Sigma-Aldrich. The reagents in solid form were dissolved in $\mathrm{ddH}_{2} \mathrm{O}$ and were then filtered using $0.22 \mu \mathrm{m}$ syringe filters (SLGP033RS, Millipore)

Plasmid circuit construction. Standard molecular biology techniques were used to construct plasmids containing arsenic or mercury-responsive genetic circuits and amplifiers. Plasmids used in this study are summarized in Supplementary Table 1, and detailed plasmid maps are provided in related figures and Supplementary Fig. 10. All primers used in this study are listed in Supplementary Table 5 and were purchased from Sigma-Aldrich. All plasmids constructed in this study have been confirmed by Sanger sequencing (Source BioScience).

For tuning the intracellular receptor densities, the constitutive promoters and the ribosome binding sites that directly drive the expression of arsR/merR were replaced via polymerase chain reaction (PCR) amplification (using Phusion highfidelity DNA polymerase (M0530L, NEB) in an Applied Biosystems Veriti Thermal Cycler). To optimize the amplifier Amp30 $0^{\mathrm{C}}$, the $\sigma^{54}$ binding site in $\mathrm{P}_{\text {hrp }}$ was replaced with a $\sigma^{54}$ consensus binding sequence (Supplementary Fig. 2) by PCR.

The rinA_p80 $\alpha$ and ECF11_987 (with the ASV tag) genes, and promoter $\mathrm{P}_{\text {rinA_p80a }}$ were synthesized by GeneArt following the BioBrick standard (http://biobricks.org). The genes were separately constructed with a terminator (BBa_B0015) via BioBrick assembly ${ }^{50}$. Various ribosome binding site sequences and a degradation tag sequence (that is, the ASV tag) were introduced to the gene by PCR. Promoter $\mathrm{P}_{\text {eff11_3726 }}$ (also named $\mathrm{P}_{e 11}$ ) was constructed by annealing oligonucleotides.

$P_{a r s R}$ variants with an extra ABS at varying distances were constructed by inverse $\mathrm{PCR}^{51}$. Genes malE and TEV were amplified from the E. coli MG1655 genome and BBa_K1319004 separately by PCR, and they were put together by Gibson Assembly ${ }^{52}$. The fused sequence was next ligated with $\mathrm{P}_{a r s \mathrm{~B}}-\mathrm{ABS} 62$ into pSB4A3 by BioBrick Assembly. The TEV protease cleavage sequence was added between the $g f p$ and $A A V$ tag sequence by inverse PCR. An extra linker sequence was then added ahead of the cleavage site by a second round of inverse PCR.

Gene expression assays and data analysis. The growth conditions for characterizing the engineered circuits are described above. GFP was used as a reporter for all sensor circuits. The $g f p$ expression was measured by BMG FLUOstar fluorometry as described above $(485 \mathrm{~nm}$ for excitation, $520 \pm 10 \mathrm{~nm}$ for emission, gain $=1,000)$. Absorbance $\left(A_{600}\right)$ was also read at the same time to determine the cell density. The fluorometry data were first processed using Omega MARS Data Analysis Software, and then were exported to Microsoft Excel 2013 and GraphPad for data analysis. The medium backgrounds of absorbance and fluorescence (fluorescence $/ A_{600}$ ) were determined from blank wells loaded with lysogeny broth media and were subtracted from the readings of other wells. The fluorescence $/ A_{600}$ at a specific time for a sample culture was determined after subtracting its triplicate-averaged counterpart of the negative control cultures (GFP-free) at the same time. The fluorescence $/ A_{600}$ after $5 \mathrm{~h}$ growth (unless indicated otherwise) post initial day dilution and induction was used as the output response of the cells in the steady state when cells were in exponential growth and the steady state assumption for protein expression is applied. Unless indicated otherwise, each sensor was tested with three or more biological replicates. The sample size (that is, $n$ ) is specified in figure legends. All the data shown are mean values and are based on the plate reader data unless otherwise indicated.

Flow cytometry assays were performed following the plate reader assays. Briefly, the cells from the 96-well plate were transferred and diluted 1:100 to another U-bottom 96-well plate (612U96, Fisher Scientific) with PBS (1X, with $2 \mathrm{mg} \mathrm{ml}^{-1}$ Kanamycin to stop translation). The transferred plate was incubated at $4^{\circ} \mathrm{C}$ for $1 \mathrm{~h}$. Cells were assayed at low flow rate until 10,000 total events were collected using Attune NxT software on an Attune NxT Flow Cytometer (equipped with Attune NxT Autosampler) using $488 \mathrm{~nm}$ excitation laser and a $530 \mathrm{~nm}$ emission filter with $30 \mathrm{~nm}$ bandpass. The flow cytometry data were analyzed using Attune NxT software and FlowJo software (v.7.6) with an appropriate gate of forward-scattering and side-scattering for all tested cultures.

Agarose gel entrapment-based sensor array assay. The sensor cells were cultured overnight as described above, and then were diluted 200 -fold into $10 \mathrm{ml}$ fresh lysogeny broth medium with appropriate antibiotics in $50 \mathrm{ml}$ Falcon centrifuge tubes. Afterward, the cells were cultured at $37^{\circ} \mathrm{C}$ with shaking at 160 r.p.m. until the $A_{600}$ reached 1.5 (after $4 \mathrm{~h} 40 \mathrm{~min}$ ). The cells were concentrated five times in PBS by centrifugation at $3,000 \mathrm{~g}$ for $5 \mathrm{~min}$ and then resuspended in PBS (K813500ML, VWR) with $250 \mathrm{\mu g} \mathrm{ml}^{-1}$ kanamycin or ampicillin. Fresh $1.25 \%$ (w/v) agarose solution was prepared in PBS (without antibiotics) for each test, and was kept at $55^{\circ} \mathrm{C}$ in a block heater to prevent solidification before use. The agarose solution was then kept at $42^{\circ} \mathrm{C}$ for $5 \mathrm{~min}$ before mixing with the resuspended cells at a $4: 1$ ratio to re-dilute the cells back to $A_{600} \approx 1.5$, making the final agarose concentration to $1 \%$, and the added antibiotics diluted to $50 \mathrm{\mu g} \mathrm{ml}^{-1}$. Next the agarose-cell mixture was quickly loaded into a 384-well microplate (781906, Greiner Bio-One) with $15 \mu \mathrm{l}$ in each well. For the arsenic induction, $\mathrm{ddH}_{2} \mathrm{O}$, $\mathrm{NaAsO}{ }_{2}$ or $\mathrm{Na}_{2} \mathrm{HAsO}_{4}$ solution was diluted 40 -fold into $\mathrm{M} 9$ medium $\left(11.28 \mathrm{gl}^{-1} \mathrm{M} 9\right.$ salts (M6030, Sigma-Aldrich), $1 \mathrm{mM}$ thiamine hydrochloride, $0.2 \%$ (w/v) casamino acids, $2 \mathrm{mM} \mathrm{MgSO}_{4}, 0.1 \mathrm{mM} \mathrm{CaCl}_{2}, 0.4 \%$ (v/v) glycerol) and $50 \mu \mathrm{l}$ of the mediuminducer mixture was added to each well. The microplate was then sealed with a sealing membrane (Z380059-1PAK, Sigma-Aldrich) and covered with a plate lid to avoid evaporation. The microplates were incubated at 37 or $25^{\circ} \mathrm{C}$, and were measured after 24 and $40 \mathrm{~h}$.

To test whether the agarose gel-entrapped sensor cell array would give false negative results under lethal antimicrobial conditions, the sensor cell array was tested under the following conditions: (1) $25 \mu \mathrm{g} \mathrm{ml}^{-1}$ chloramphenicol, (2) $\mathrm{pH}=3.5$ (via addition of acetic acid, 338826, Honeywell Research Chemicals), (3) $\mathrm{pH}=11.4$ (via addition of sodium hydroxide, 71690, Sigma-Aldrich) and (4) $1 \mathrm{mM}$ or $3 \mathrm{mM} \mathrm{CuSO}_{4}$.

A variety of media were tested for their abilities to maximize the sensors responses to arsenate. PBS was replaced by $0.9 \% \mathrm{NaCl}$ solution in these experiments to resuspend cells and to make agarose solution. M9 medium was optimized by reducing both $\mathrm{Na}_{2} \mathrm{HPO}_{4}$ and $\mathrm{KH}_{2} \mathrm{PO}_{4}$ levels to $1 / 4,1 / 16$ and $1 / 64$ of the original concentrations, or by replacing the phosphate-based buffer with a TrisHCl-based buffer ( $10.992 \mathrm{gl}^{-1}$ Tris- $\left.\mathrm{HCl}\right)$. The $\mathrm{pH}$ of M9 (Tris- $\left.\mathrm{HCl}\right)$ medium was adjusted to $\mathrm{pH}=7$ via addition of $1 \mathrm{M} \mathrm{NaOH}$ solution. Since agarose-entrapped cells did not grow when glucose was used as the carbon source (Supplementary Fig. 7m,n), we used a modified MOPS EZ Rich Defined Medium (M2105, TEKnova) where glucose was replaced by glycerol $(0.2 \%(\mathrm{v} / \mathrm{v}))$. The MOPS medium was further optimized by reducing the $\mathrm{K}_{2} \mathrm{HPO}_{4}$ level to $3 / 4,1 / 2$ and $1 / 4$ of its original concentration.

The fluorescent signals of the microplates were measured by a BMG FLUOstar fluorometer, and photographs were taken by a Bio-Rad Gel Doc XR+ system with filter 1 and SYBR Green mode. The images were acquired by Image Lab software at 600 dots-per-inch resolution. Each image was first adjusted using Photoshop CS3 software to reduce background by subtracting the brightness of negative controls, and then was adjusted to increase brightness and contrast. To prepare for cell phone imaging, the microplates were placed onto the surface of a Safe Imager (S37102, Invitrogen) blue-light transilluminator and were covered with an amber filter in a dark environment. A cell phone (OPPO X9000) was used to acquire the images with the build-in high dynamic range (HDR) setting and the images were then adjusted to increase brightness and contrast using Photoshop CS3 software.

Groundwater samples preparation. Groundwater samples were collected from wells at a local village in Khulna, Bangladesh. The samples were filtered through $0.22 \mu \mathrm{m}$ syringe filters before arsenic quantification using inductively coupled plasma mass spectrometry (ICP-MS). The quantification was carried out by L. Eades, the ICP-MS instrument technician in the School of Chemistry of Edinburgh University. The determined arsenic quantities were reported in Supplementary Table 4 . For functionality test of the sensor array, the quantified samples were mixed with $2 \times$ MOPS (with $1 / 4 \mathrm{PO}_{4}{ }^{3-}$ and $0.2 \%(\mathrm{v} / \mathrm{v})$ glycerol after mixing) at the ratio of $1: 1$, and were then incubated with the sensor array at $37^{\circ} \mathrm{C}$.

Microfluidic encapsulation-based sensor array assay. The microfluidic device is composed of two polydimethylsiloxane (PDMS) layers, a control and a flow. The molds for the two layers were fabricated using standard photolithography techniques. A 4' silicon wafer was coated with $30 \mu \mathrm{m}$ GM1050 SU-8 and $14 \mu \mathrm{m}$ AZ9260 photoresist for the control and the flow mold, respectively. After exposure and development, the flow mold was placed at $160^{\circ} \mathrm{C}$ for $2 \mathrm{~h}$ to round the channels Devices were cast in PDMS using multilayer soft lithography. PDMS was prepared at a 20:1 ratio and spin-coated onto the flow mold at 3,000 r.p.m. for $1 \mathrm{~min}$ using a SCS G3P-8 Spin Coater. For the control, PDMS at a ratio of 5:1 was poured onto the control mold. Both layers were cured at $80^{\circ} \mathrm{C}$ for $30 \mathrm{~min}$ and successively aligned. After the alignment, the flow and the control layers were bonded at $80^{\circ} \mathrm{C}$ for $1.5 \mathrm{~h}$.

The cells were spotted on an epoxysilane (3-glycidoxypropyldimethoxymethylsilane 97\%, AC216545000, Acros Organic) functionalized glass slide (Supplementary Fig. 6i). After overnight growth in lysogeny broth medium 
at $37^{\circ} \mathrm{C}$ with 200 r.p.m. shaking, the cells were centrifuged for $5 \mathrm{~min}$ at $1,781 \mathrm{~g}$ and the cell pellet was resuspended in $150 \mu$ lysogeny broth medium with $10 \%$ glycerol. To generate the pattern, the cells were spotted using a microarray robot (Qarray2, Genetix) and a $1.7 \mathrm{nl}$ delivery volume pin (946MP2B, Arrayit). The microfluidic device was then aligned and bonded to the spotted glass slide for $1 \mathrm{~h}$ at $37^{\circ} \mathrm{C}$.

After bonding, the control lines of the device were primed with PBS at $5 \mathrm{ps}$ using tygon tubings. Once the control lines were filled up the pressure was increased to $10 \mathrm{psi}$ and the flow pressure was set to $2 \mathrm{psi}$. LabVIEW 2010 software was used to facilitate continuous cell culturing and arsenic induction in the device. The culturing program comprises three steps: (1) flowing lysogeny broth medium into the flow channels (keeping the chamber valves closed) for $10 \mathrm{~min}$ (2) incubating the cells (keeping the sandwich valves closed and the chamber valve opened) with the medium for $45 \mathrm{~min}$ and (3) washing with lysis buffer containing $30 \mathrm{mM}$ of $\mathrm{NaOH}$ (06203, Sigma-Aldrich) and 12\% SDS (L3771, Sigma-Aldrich) (with chamber valve closed) for $10 \mathrm{~min}$ (Supplementary Fig. 6i). After overnight growth, the inducer $\mathrm{NaAsO}_{2}$ (35000-1L-R, Fluka), diluted in lysogeny broth medium, was flowed through the device. A temperature controlled glass plate (H401-NIKON-Ti_SR_Glass / 401_T_CONTROLLER, Okolab) was used to keep the microfluidic device at $37^{\circ} \mathrm{C}$ during all experiments.

Fluorescent images were acquired by a Nikon ECLIPSE Ti automated microscope (using NIS-Elements AR 4.30.02 (build 1053) software) with a lightemitting diode fluorescent excitation system and a Hamamatsu ORCA-Flash 4.0 camera using a $\times 40$ magnification objective (SPlan Fluor, ELWD $\times 40 / 0.60$, $\infty / 0.2$, WD 3.6-2.8, Nikon). A USB fluorescence microscope (AM4113T-GFBW, Dino-Lite) with FITC fluorescent filter was used to acquire fluorescent images at low magnification $(\times 10)$ using DinoCapture 2.0 software. Nine subsections of the area of interest were taken and successively stitched together using Fiji software (ImageJ with Grid/Collection Stitching plugin). All the images were adjusted using Photoshop CS3 to increase brightness and contrast. Finally, a cell phone (iPhone 5) was used to take images of the device after the induction. A bandpass filter (centered at $530 \mathrm{~nm}$ with a $40 \mathrm{~nm}$ bandwidth) was placed in front of the camera to filtrate the excitation wavelength and a blue light-emitting diode was used for illumination. The cell phone images were adjusted using Photoshop CS3 to reduce background and to increase brightness and contrast.

Calculation of sensor detection limit. The LOD is the lowest analyte concentration that can probably be reliably distinguished from the basal signal and at which detection is feasible ${ }^{53}$. The basal signal can be described as limit of blank (LOB):

$$
\mathrm{LOB}=\mu_{\mathrm{B}}+1.645 \mathrm{~s} \cdot \mathrm{d}_{\cdot \mathrm{B}}
$$

where LOB is estimated by measuring replicates of a blank sample containing no analyte and calculating the mean $\left(\mu_{\mathrm{B}}\right)$ and the standard deviation (s.d. $\left.{ }_{\mathrm{B}}\right)$. Assuming a Gaussian distribution of the raw analytical signals from blank samples, the LOB represents $95 \%$ of the observed values. The LOD is determined by the measured LOB and test replicates with known low concentration of the analyte. It can be described as

$$
\text { Output }_{\mathrm{LOD}}=\mathrm{LOB}+1.645 \mathrm{~s} \cdot \mathrm{d}_{\mathrm{L}}
$$

where s.d. ${ }_{\mathrm{L}}$ represents the standard deviation of measured replicates with a low concentration of analyte. Assuming a Gaussian distribution again for the low concentration samples, $95 \%$ of values will exceed the LOB. When the mean $\left(\mu_{\mathrm{L}}\right)$ value of the measured replicates equals to Output $\mathrm{LD}_{\mathrm{LD}}$, the concentration of the analyte will be the LOD. Therefore, we estimated the LOD of our sensors when

$$
\mu_{\mathrm{L}}-\left(\mathrm{LOB}+1.645 \mathrm{~s} . \mathrm{d}_{\mathrm{L}}\right) \approx 0
$$

Calculation of noise factor and signal-to-noise ratio (SNR). Noise factor and SNR were calculated as described in a previous study ${ }^{21}$. Briefly, the noise factor (NF) is the ratio between the SNR of input and output: $\mathrm{NF}=\mathrm{SNR}_{\mathrm{IN}} / \mathrm{SNR}_{\mathrm{OUT}}{ }^{21}$. The SNR is defined by the ratio of the sample's mean fluorescence to its standard deviation at single cell level: $\mathrm{SNR}=\mu / \sigma^{21}$. The $\mathrm{SNR}_{\mathrm{IN}}$ is the $\mathrm{SNR}$ at the device input (that is, sensor output without amplification), while the $\mathrm{SNR}_{\mathrm{OUT}}$ is the $\mathrm{SNR}$ at the device output (that is, sensor output after amplification). The values were calculated from single cell flow cytometry assays (Supplementary Figs. 1,2 and 8).

Mathematical modeling and data fitting. Biochemical models were developed for individual transcription factor receptor modules to abstract their ligand-dependen dose-response behaviors. The ordinary differential equation-based deterministic model was used for accurately modeling the gene regulation and expression across the full input or output range of the sensor systems $\mathrm{s}^{21,54}$. For inducible sensor promoters used (arsR- $\mathrm{P}_{a r s R}$ and merR- $\mathrm{P}_{\text {mer } T}$ promoters), the promoter $\mathrm{P}_{R}\left(\mathrm{P}_{a r s R} / \mathrm{P}_{\text {merT } T}\right)$ is negatively regulated by its constitutively expressed repressor $\mathrm{R}$ (ArsR/MerR) and is responsive to exogenous inducer I (arsenic/mercury) to activate transcription of downstream reporter gene $G$. The output gene expression is modeled by ref. ${ }^{54}$

$$
\mathrm{d}[G] / \mathrm{d} t=\alpha k+k[I]^{n} /\left(K_{M}^{n}+[I]^{n}\right)-d[G]
$$

where $\alpha k$ is the basal constitutive activity of the promoter, $k[I]^{n} /\left(K_{M}^{n}+[I]^{n}\right)$ is the activity due to cooperative transcription activation and $d[G]$ is the constitutive degradation activity of protein $G . K_{M}$ and $n$ are the Hill constant and coefficient, respectively, relating to the promoter-regulator/inducer interaction, $k$ is the maximum expression rate due to induction and $\alpha$ is a constant relating to the promoter basal activity level due to leakage $(0 \leq \alpha<1)$, and $d$ is the degradation rate of $G$.

The steady-state solution of equation (S4) is given by

$$
f([I])=[G]_{S S}=\alpha k+k[I]^{n} /\left(K_{M}^{n}+[I]^{n}\right)
$$

where $k=k_{1} / d$ represents the maximum expression level due to induction. Equation (S5) gives the reporter protein level at steady state for the inducible promoter $\mathrm{P}_{R}$ and is also the transfer function of $\mathrm{P}_{R}$. We used this transfer function to fit the characterization data of the arsenic and mercury inducible promoters and engineered sensor systems using GraphPad software. The best fit parameters and coefficients (with 95\% confidence bounds) are listed in Supplementary Table 3 and the parameterized transfer functions are plotted in Figs. 2b,d,3b,c and 4a,b and Supplementary Figs. 4a and 6a, respectively, against their experimental data. Supplementary Fig. 11 shows the linear correlation between predicted and experimentally characterized responses of the sensors.

A simple linear mathematical formula was used to model the input-output relationship in the linear amplification dynamic range of the one-layer amplifier system:

$$
y=k(x-b)
$$

where the slope (also known as amplification gain) is $k=\Delta y / \Delta x$, and $b$ is the constant related to the $y$ intercept when $x=0$. The parameterized transfer functions are plotted in Fig. 2e against their experimental data.

Reporting Summary. Further information on research design is available in the Nature Research Reporting Summary linked to this article.

\section{Data availability}

All data and plasmids supporting the findings are available from the corresponding author upon reasonable request.

\section{References}

50. Shetty, R. P., Endy, D. \& Knight, T. F. Engineering BioBrick vectors from BioBrick parts. J. Biol. Eng. 2, 5 (2008).

51. Ochman, H., Gerber, A. S. \& Hartl, D. L. Genetic applications of an inverse polymerase chain reaction. Genetics 120, 621-623 (1988).

52. Gibson, D. G. et al. Enzymatic assembly of DNA molecules up to several hundred kilobases. Nat. Methods 6, 343-345 (2009).

53. Armbruster, D. A. \& Pry, T. Limit of blank, limit of detection and limit of quantitation. Clin. Biochem. Rev. 29, S49-S52 (2008).

54. Wang, B., Kitney, R. I., Joly, N. \& Buck, M. Engineering modular and orthogonal genetic logic gates for robust digital-like synthetic biology. Nat. Commun. 2, 508 (2011). 


\section{Reporting Summary}

Nature Research wishes to improve the reproducibility of the work that we publish. This form provides structure for consistency and transparency in reporting. For further information on Nature Research policies, see Authors \& Referees and the Editorial Policy Checklist.

\section{Statistical parameters}

When statistical analyses are reported, confirm that the following items are present in the relevant location (e.g. figure legend, table legend, main text, or Methods section).

n/a Confirmed

$\bigotimes$ The exact sample size $(n)$ for each experimental group/condition, given as a discrete number and unit of measurement

$\searrow$ An indication of whether measurements were taken from distinct samples or whether the same sample was measured repeatedly

$\square$ The statistical test(s) used AND whether they are one- or two-sided

$\bigotimes \square$ Only common tests should be described solely by name; describe more complex techniques in the Methods section.

$\bigotimes$ A description of all covariates tested

\ A description of any assumptions or corrections, such as tests of normality and adjustment for multiple comparisons

$\square \bigotimes$ A full description of the statistics including central tendency (e.g. means) or other basic estimates (e.g. regression coefficient) AND variation (e.g. standard deviation) or associated estimates of uncertainty (e.g. confidence intervals)

For null hypothesis testing, the test statistic (e.g. $F, t, r$ ) with confidence intervals, effect sizes, degrees of freedom and $P$ value noted Give $P$ values as exact values whenever suitable.

Х $\square$ For Bayesian analysis, information on the choice of priors and Markov chain Monte Carlo settings

Х $\square$ For hierarchical and complex designs, identification of the appropriate level for tests and full reporting of outcomes

\ Estimates of effect sizes (e.g. Cohen's $d$, Pearson's $r$ ), indicating how they were calculated

$\square$ Clearly defined error bars

$\bigotimes$ State explicitly what error bars represent (e.g. SD, SE, CI)

Our web collection on statistics for biologists may be useful.

\section{Software and code}

Policy information about availability of computer code

Data collection

Omega MARS 3.20 R2, Attune NxT Software v2.5, Image Lab software v5.2.1, LabVIEW2010, NIS-Elements AR 4.30.02 (build 1053), DinoCapture 2.0.

Data analysis

Microsoft Excel 2013, GraphPad Prism 6.01, Omega MARS 3.20 R2, Attune NxT Software v2.5, FlowJo 7.6.1, Photoshop CS3, Fiji (ImageJ with Grid/Collection Stitching plugin).

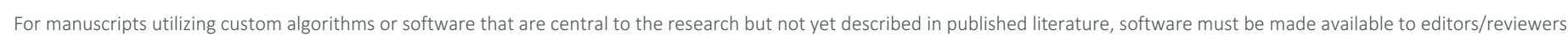

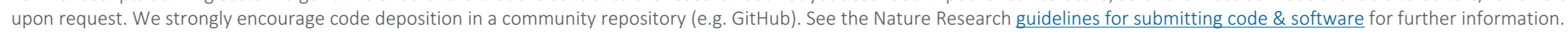

\section{Data}

Policy information about availability of data

All manuscripts must include a data availability statement. This statement should provide the following information, where applicable:

- Accession codes, unique identifiers, or web links for publicly available datasets

- A list of figures that have associated raw data

- A description of any restrictions on data availability

All data and plasmids supporting the findings are available from the corresponding author upon reasonable request. 


\section{Field-specific reporting}

Please select the best fit for your research. If you are not sure, read the appropriate sections before making your selection.

$\bigotimes$ Life sciences $\quad \square$ Behavioural \& social sciences $\square$ Ecological, evolutionary \& environmental sciences

For a reference copy of the document with all sections, see nature.com/authors/policies/ReportingSummary-flat.pdf

\section{Life sciences study design}

All studies must disclose on these points even when the disclosure is negative.

Sample size Sample sizes in terms of number of replicates are standard in the field, and are described along with Figures. No statistical methods were used to determine the sample sizes.

Data exclusions No data were excluded from the analyses.

Replication All experiments were performed in three or more replicates. All experiments were biological repeats unless stated otherwise. All attempts at replication were successful.

Randomization Each biological replicate of a bacterial culture was inoculated from a single colony, which was randomly chosen from an agar plate.

Blinding No blinding was involved as it was not relevant to this study. No animal or human research participants were utilized and all samples were processed in parallel.

\section{Reporting for specific materials, systems and methods}

Materials \& experimental systems

$\mathrm{n} / \mathrm{a}$ Involved in the study

$\searrow \square$ Unique biological materials

Х $\square$ Antibodies

$\backslash \square$ Eukaryotic cell lines

Х $\square$ Palaeontology

Х Animals and other organisms

$\searrow \square$ Human research participants

\begin{tabular}{l|l}
\multicolumn{2}{l}{ Methods } \\
\hline n/a Involved in the study \\
$\square$ ChIP-seq \\
$\square$ Mrlow cytometry
\end{tabular}

Flow Cytometry

Plots

Confirm that:

Х The axis labels state the marker and fluorochrome used (e.g. CD4-FITC).

$\bigotimes$ The axis scales are clearly visible. Include numbers along axes only for bottom left plot of group (a 'group' is an analysis of identical markers).

$\square$ All plots are contour plots with outliers or pseudocolor plots.

$\square$ A numerical value for number of cells or percentage (with statistics) is provided.

\section{Methodology}

Sample preparation

Bacterial cells from the 96-well plate culture were transferred and diluted $1: 100$ to another U-bottom 96-well plate with PBS (1 $\times$, with $2 \mathrm{mg} / \mathrm{ml}$ Kanamycin to stop translation). The transferred plate was incubated at $4^{\circ} \mathrm{C}$ for $1 \mathrm{~h}$ before the flow cytometry assay.

Instrument

Attune NxT Flow Cytometer and Attune NxT Autosampler.

Software

Attune NxT Software v2.5, FlowJo 7.6.1 and Microsoft Excel 2013.

Cell population abundance 
Tick this box to confirm that a figure exemplifying the gating strategy is provided in the Supplementary Information. 